\title{
A review of antennas and propagation for MIMO wireless communications
}

Michael A. Jensen

jensen@byu.edu

Jon W. Wallace

wall@ieee.org

Follow this and additional works at: https://scholarsarchive.byu.edu/facpub

Part of the Electrical and Computer Engineering Commons

\section{Original Publication Citation}

Jensen, M. A., and J. W. Wallace. A Review of Antennas and Propagation for MIMO Wireless Communications." Antennas and Propagation, IEEE Transactions on 52.11 (24): 281-24

\section{BYU ScholarsArchive Citation}

Jensen, Michael A. and Wallace, Jon W., "A review of antennas and propagation for MIMO wireless communications" (2004). Faculty Publications. 409.

https://scholarsarchive.byu.edu/facpub/409 


\title{
A Review of Antennas and Propagation for MIMO Wireless Communications
}

\author{
Michael A. Jensen, Senior Member, IEEE, and Jon W. Wallace, Member, IEEE
}

Invited Paper

\begin{abstract}
Multiple-input-multiple-output (MIMO) wireless systems use multiple antenna elements at transmit and receive to offer improved capacity over single antenna topologies in multipath channels. In such systems, the antenna properties as well as the multipath channel characteristics play a key role in determining communication performance. This paper reviews recent research findings concerning antennas and propagation in MIMO systems. Issues considered include channel capacity computation, channel measurement and modeling approaches, and the impact of antenna element properties and array configuration on system performance. Throughout the discussion, outstanding research questions in these areas are highlighted.
\end{abstract}

Index Terms-Antenna arrays, multiple-input-multiple-output (MIMO) systems, propagation.

\section{INTRODUCTION}

$\mathbf{M}$ ULTIPLE-INPUT-MULTIPLE-OUTPUT (MIMO) wireless systems, characterized by multiple antenna elements at the transmitter and receiver, have demonstrated the potential for increased capacity in rich multipath environments [1]-[4]. Such systems operate by exploiting the spatial properties of the multipath channel, thereby offering a new dimension which can be used to enable enhanced communication performance.

While coding and signal processing are key elements to successful implementation of a MIMO system, the propagation channel and antenna design represent major parameters that ultimately impact system performance. As a result, considerable research has been devoted recently to these two areas. For example, assessing the potential of MIMO systems requires a new level of understanding concerning multipath channel characteristics. Furthermore, while we have extensive information concerning the behavior of antenna diversity in multipath channels [5], recent activity surrounding MIMO communications has exposed new issues related to the impact of antenna properties and array configuration on system performance.

The goal of this paper is to provide both a brief tutorial on MIMO systems suitable for those involved in antenna and propagation research as well as a review of some of the significant

Manuscript received May 9, 2003; revised October 21, 2003. This work was supported by the National Science Foundation under Wireless Initiative Grant CCR 99-79452 and Information Technology Research Grant CCR-0081476.

The authors are with the Department of Electrical and Computer Engineering, Brigham Young University, Provo, UT 84602 USA (e-mail: jensen@ee.byu.edu).

Digital Object Identifier 10.1109/TAP.2004.835272

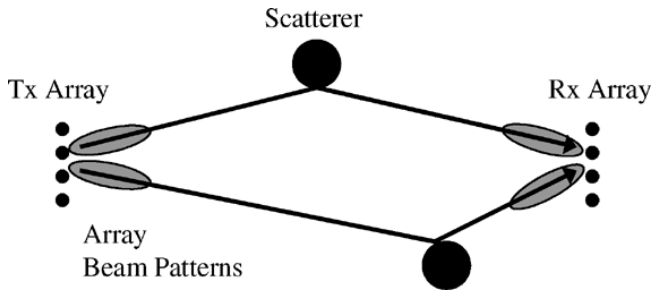

Fig. 1. Simple multipath propagation environment showing two paths between transmit and receive. The arrays are capable of resolving the individual multipaths, enabling increased data throughput.

findings related to antennas and propagation within the context of this communication strategy [6], [7]. However, as with any emerging research arena, the volume of material that has appeared is too large to summarize in one brief paper. As a result, this summary will focus on a subset of these issues, believed by the authors to be key considerations that may spawn additional research activities within the antennas and propagation community. Related findings from multiple independent studies will be synthesized to provide a broader perspective of the state-of-the art in this exciting research field.

The remainder of the paper is divided into three main parts. First, Section II provides a tutorial on MIMO systems and demonstrates how the use of multiple antennas can lead to increased capacity bounds. Section III then discusses different measurement and modeling approaches for multipath MIMO channels. Finally, Section IV focuses on the impact of antenna parameters on MIMO system performance. Throughout the discussion, possible future research activities are highlighted.

\section{MIMO SYSTEM TUTORIAL}

Before embarking on a detailed discussion of recent research, it is useful to establish some notation, provide a common framework for discussion, and present tutorial material relating to MIMO communication. Throughout this paper, matrices and column vectors are represented as boldface uppercase and lowercase letters, respectively, $H_{i j}$ is the element occupying $i$ th row and $j$ th column of the matrix $\mathbf{H}$, and $h_{i}$ is the $i$ th element of the vector $\mathbf{h}$.

\section{A. MIMO Multipath Communication}

The concept of using spatial (array) processing to enhance communication performance has been well explored. For example, consider a wireless communication node equipped 


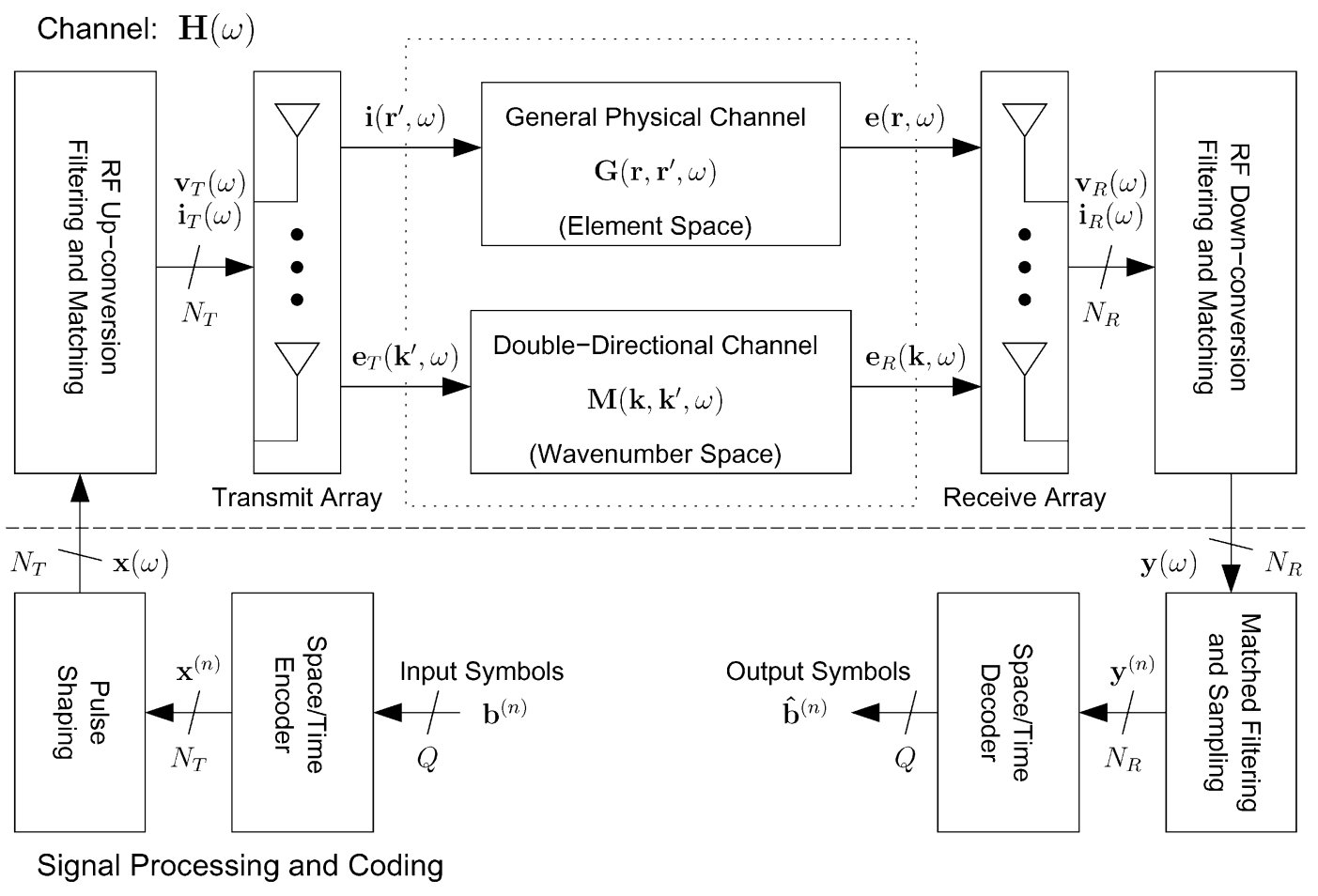

Fig. 2. Block diagram of a generic MIMO wireless system.

with an $N$-element antenna array that must send data to $N$ distinct users. Using traditional beamforming (or null-steering), the system can synthesize an array response to transmit data to a single user while placing nulls on the remaining $N-1$ users. Therefore, by synthesizing a unique pattern for each user and encoding a unique data stream on each pattern, the system can simultaneously communicate with all users with a spectral occupancy equal to that of a single data stream.

This same principle can be used in a point-to-point multipath channel. For example, consider the scenario depicted in Fig. 1 which shows two propagation paths between a transmitter and receiver. If the arrays can resolve the two multipaths, the system can encode a unique data stream on each propagation path, resulting in an increase in communication capacity without an increase in required bandwidth. As typical wireless channels consist of many different closely spaced (in angle) paths, resolution of individual multipaths is often not possible. Therefore, MIMO implementations must use more advanced array signal processing to exploit the channel spatial resources. Before discussing these concepts in more detail, however, we first define a model for the MIMO communication system to facilitate the presentation.

\section{B. Communication System Model}

A general model of a MIMO communication system is represented in Fig. 2. For simplicity, the channel is assumed time invariant over the interval of a transmission block. The figure is divided into 1) signal processing and coding (bottom) and 2) the channel (top). The radio frequency (RF) components are included in the channel since they influence the end-to-end transfer function.

In this system, a set of $Q$ independent data streams represented by the symbol vector $\mathbf{b}^{(n)}$ ( $n$ is a time index) are encoded into $N_{T}$ discrete-time complex baseband streams $\mathbf{x}^{(n)}$ at the transmitter. The coding can distribute the input symbols over the $N_{T}$ outputs (space) and/or over samples (time). The pulse-shaping block converts the discrete-time samples into continuous-time baseband waveforms $\mathbf{x}(\omega)$ ( $\omega$ is frequency) and feeds them to the $N_{T}$ channel inputs (RF chains and antennas). The channel $\mathbf{H}(\omega)$ combines the input signals to obtain the $N_{R}$ element output (receive) waveform vector $\mathbf{y}(\omega)$. The matched filter then produces the discrete-time baseband sample stream $\mathbf{y}^{(n)}$, and the space/time decoder generates estimates of the $Q$ transmitted streams $\hat{\mathbf{b}}^{(n)}$.

For linear channel elements, the MIMO channel input-output relationship may be written as

$$
\underset{N_{R} \times 1}{\mathbf{y}(\omega)}=\underset{N_{R} \times N_{T}}{\mathbf{H}(\omega)} \underset{N_{T} \times 1}{\mathbf{x}(\omega)}+\underset{N_{R} \times 1}{\eta(\omega)}
$$

where $\eta(\omega)$ is additive noise produced by the channel (interference plus noise from the RF front end) and the matrix dimensions are as specified. Each element $H_{i j}(\omega)$ represents the transfer function between the $j$ th transmit and $i$ th receive antenna. Since the transmit vector is projected onto $\mathbf{H}(\omega)$ in (1), the number of independent data streams $(Q)$ that can be supported must be at most equal to the rank of $\mathbf{H}(\omega)$. More generally, the properties of $\mathbf{H}(\omega)$, such as the distribution of its singular values, determine the performance potential for the MIMO system. Factors such as antenna impedance matching, array size and configuration, element pattern and polarization properties, mutual coupling, and multipath propagation characteristics influence these properties. Therefore, poor design of system components or incorrect assumptions about the channel could lead to drastic reduction in system performance.

For convenience, we will usually drop the frequency dependence $\omega$ and consider narrowband communication, which 
is justified when the channel response is constant over the system bandwidth (flat fading) or when signals are divided into narrowband frequency bins and processed independently. This highlights the effect of the spatial dimension, a unique factor of MIMO communications, and ignores the complexity of the wide-band channel response.

\section{MIMO Beamforming}

We now consider the situation where the transmitter and receiver know the channel matrix $\mathbf{H}$. Let the unencoded $N_{T} \times 1$ transmit vector in the waveform domain be denoted as $\mathbf{x}^{\prime}$. Using the singular value decomposition (SVD) $\mathbf{H}=\mathbf{U S V}^{H}$, where $\{\cdot\}^{H}$ denotes a conjugate transpose, we encode the transmit vector as $\mathbf{x}=\mathbf{V} \mathbf{x}^{\prime}$. Since each element of $\mathbf{x}^{\prime}$ multiplies the corresponding column of $\mathbf{V}$, this operation suggests that each column of $\mathbf{V}$ represents array weights for each signal stream. The receiver performs the operation $\mathbf{y}^{\prime}=\mathbf{U}^{H} \mathbf{y}$, indicating that each row of $\mathbf{U}^{H}$ (column of $\mathbf{U}^{*}$ ) represents the receive array weights for each stream. Because $\mathbf{U}$ and $\mathbf{V}$ are unitary, from (1) we obtain

$$
\mathbf{y}^{\prime}=\mathbf{U}^{H} \mathbf{y}=\mathbf{S} \mathbf{x}^{\prime}+\boldsymbol{\eta}^{\prime}
$$

where $\boldsymbol{\eta}^{\prime}=\mathbf{U}^{H} \boldsymbol{\eta}$. Since the matrix $\mathbf{S}$ of singular values is diagonal, (2) indicates that $\mathbf{y}^{\prime}$ is a scaled version of the transmit vector $\mathbf{x}^{\prime}$ corrupted by additive noise. Therefore, beamforming using the singular vectors of $\mathbf{H}$ as array weights has produced eigenpatterns that create independent (spatially orthogonal) parallel communication channels in the multipath environment (see [8] for detailed discussion). Note that each of these eigenchannels in general exploits all multipath components. Also, in rank deficient channels, the number $Q$ of independent streams should correspond to the number of singular values that are above the noise floor. In this case, only the first $Q$ columns of $\mathbf{U}, \mathbf{S}$, and $\mathrm{V}$ should be used in the processing and analysis. In practice, the fraction of power allocated to each eigenchannel should be chosen to optimize the capacity, a topic considered briefly in Section II-D.

Under certain circumstances, these eigenpatterns can be directly related to the propagation scenario. Consider the example of ten vertical dipoles along the $x$ axis with a uniform spacing of $\lambda / 2$, where $\lambda$ is the free-space wavelength. Three "clusters" of plane waves with propagation vectors in the horizontal plane arrive at the receiver, with each cluster consisting of a central plane wave with two additional waves, $10^{\circ}$ either side of the center, with field strengths 0.7 times the central wave strength. The clusters are centered at $\phi=0^{\circ}$ (field strength $=1$ ), $\phi=45^{\circ}$ (field strength $=0.7$ ), $\phi=260^{\circ}$ (field strength $=0.85$ ). The receive eigenpatterns corresponding to the three largest singular values, shown in Fig. 3(a), are focused on these clusters. Fig. 3(b) shows the eigenpatterns that result when a three-element array is used for the same channel. Here, it becomes clear that if the array cannot resolve the multipaths, then the eigenpatterns achieve a superposition of the waves in an effort to maximize performance.

Because the eigenchannels are the most efficient "basis" for communicating over the channel, use of the SVD encoding/decoding coupled with optimal power allocation over the eigen-
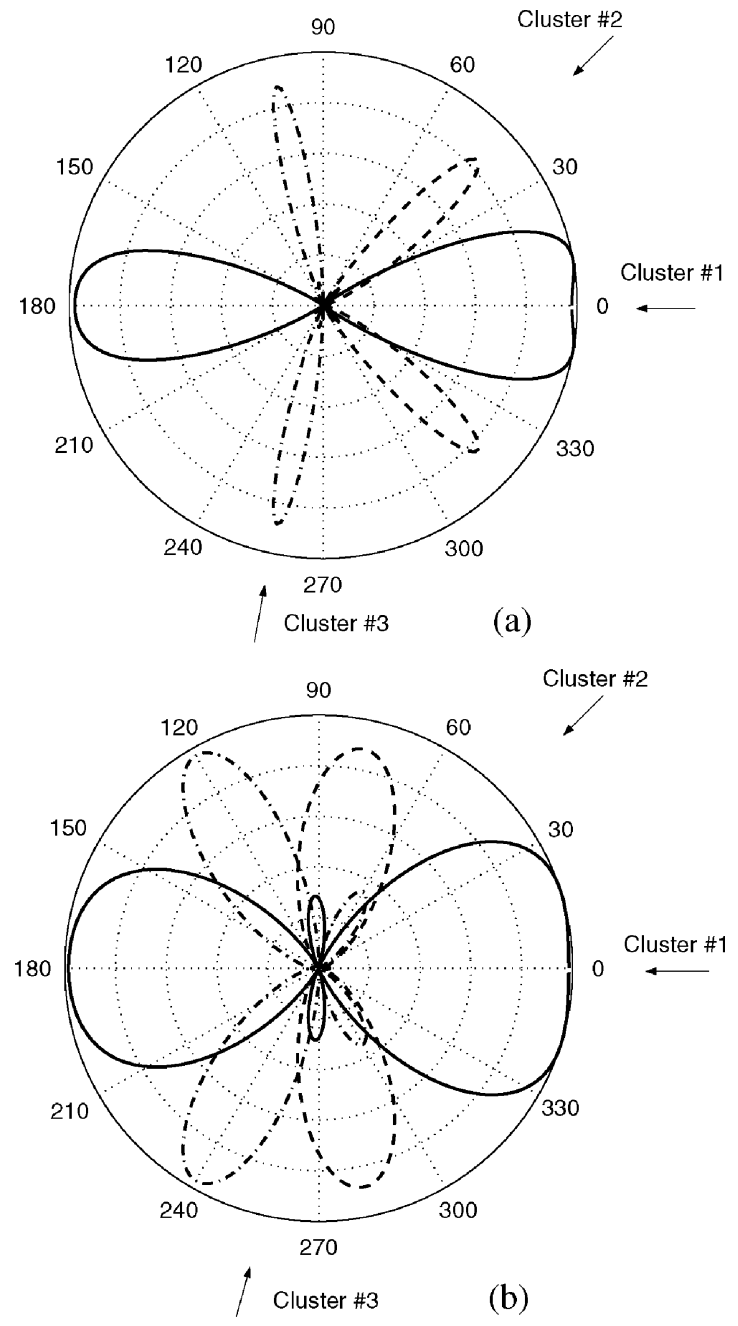

Fig. 3. Dominant three receive eigenpatterns created for a channel consisting of three plane wave clusters centered at the angles indicated by the arrows: (a) ten-element array and (b) three-element array.

channels provides optimal communication performance. However, a simpler strategy, often providing excellent performance, is simply to communicate using the individual element patterns, one for each data stream. This accomplished by directly applying each element of $\mathrm{x}^{\prime}$ to each antenna. If $N_{R} \geq N_{T}$, then the maximum likelihood estimate of the vector $\mathbf{x}^{\prime}$ can be obtained (under the assumption of spatially white Gaussian noise) from

$$
\hat{\mathbf{x}}^{\prime}=\mathbf{H}^{+} \mathbf{y}
$$

where $\{\cdot\}^{+}$represents a pseudo-inverse operation. This is the basic principle behind the well-publicized VBLAST algorithm [9], [10]. This scheme is attractive as it does not require the transmitter to know $\mathbf{H}$, which typically must be fed back from the receiver where channel estimation is performed. The key liability with this uninformed transmitter approach, however, is that if the propagation environment creates a rank-deficient channel matrix $\mathbf{H}$, the performance deteriorates rapidly. Compromise techniques have also been proposed wherein the transmit beamforming is accomplished using long-term channel statistics rather than deterministic channel knowledge. Certainly, choice of an approach will depend on anticipated 
channel characteristics coupled with complexity/performance constraints.

\section{MIMO System Capacity}

It is convenient to define a single metric that captures both the signal-to-noise ratio (SNR) and multipath spatial characteristics to represent the quality of the MIMO channel. The Shannon channel capacity [11] has emerged as the de facto measure of choice for MIMO system analysis [12]. Intuitively, this quantity represents the highest error-free MIMO transmission rate for a given transfer matrix under optimal space/time-coding and modulation.

For the waveform channel in (1), capacity represents the transmission rate per Hertz of bandwidth (bits/s/Hz). However, if pulse shaping and matched filtering are included, a discrete-time channel relating $\mathbf{x}^{(n)}$ and $\mathbf{y}^{(n)}$ results, the capacity of which represents the bits conveyed per transmission time slot (bits/use). For optimal pulse shaping and matched filtering, the continuous and discrete domains are related through Nyquist sampling [11]. These conditions lead to $C$ bits/use for an underlying waveform channel with $C$ bits/s/Hz. Therefore, capacity expressions below are valid for complex baseband channels in both discrete and continuous time provided that proper signal/noise power definitions are used.

To see how parallel spatial channels can increase capacity, consider the simple case of $Q$ uncoupled transmission lines. If only one transmission line is used to send data, the Shannon channel capacity will be [11]

$$
C_{1}=\log _{2}(1+\rho)
$$

where $\rho$ is the receiver SNR. If the transmit power is instead equally divided among the lines, the capacity becomes

$$
C_{Q}=\sum_{q=1}^{Q} \log _{2}\left(1+\frac{\rho}{Q}\right)=Q \log _{2}\left(1+\frac{\rho}{Q}\right)
$$

where we have assumed equal receiver noise. The increase in capacity is observed by recognizing that the multiplication by $Q$ outside the logarithm more than offsets the division by $Q$ inside the logarithm.

For the MIMO system described by (1), we assume that $\boldsymbol{\eta}(\omega)$ has independent Gaussian-distributed elements with equal variance $\sigma^{2}$. For a transmit vector $\mathbf{x}(\omega)$ whose elements are complex Gaussian-distributed random variables, the expression for channel capacity is

$$
\begin{aligned}
C & =\max _{\left\{\mathbf{R}_{x}: \operatorname{Tr}\left(\mathbf{R}_{x}\right) \leq P_{T}\right\}} \log _{2}\left|\mathbf{I}+\frac{\mathbf{H R}_{x} \mathbf{H}^{H}}{\sigma^{2}}\right| \\
& =\max _{\left\{\mathbf{R}_{x}: \operatorname{Tr}\left(\mathbf{R}_{x}\right) \leq P_{T}\right\}} \sum_{i} \log _{2}\left(1+\frac{\Lambda_{i i}}{\sigma^{2}}\right)
\end{aligned}
$$

where $\mathbf{H R}_{x} \mathbf{H}^{H}=\boldsymbol{\xi} \boldsymbol{\Lambda} \boldsymbol{\xi}^{H}$ is an eigenvector decomposition (EVD), $\mathbf{I}$ is the identity matrix, $|\cdot|$ is the determinant, $\operatorname{Tr}(\cdot)$ is the trace, and $P_{T}$ is the total transmit power. The diagonal elements of the transmit covariance $\mathbf{R}_{x}=\mathrm{E}\left\{\mathbf{x x}^{H}\right\}$, where $\mathrm{E}\{\cdot\}$ is the expectation, represent the transmit power from each antenna, and therefore the constraint $\operatorname{Tr}\left(\mathbf{R}_{x}\right) \leq P_{T}$ limits the total transmit power. The off-diagonal elements of $\mathbf{R}_{x}$ represent the correlation between the transmitted signal streams, with increased correlation resulting in decreased capacity [reduction in some values $\Lambda_{i i}$ in (7)]. Note that the term $\mathbf{H R}_{x} \mathbf{H}^{H}=\mathrm{E}\left\{\mathbf{H x x}{ }^{H} \mathbf{H}^{H}\right\}$ represents the covariance of the received signal in the absence of noise, so that the eigenvalue $\Lambda_{i i}$ represents the received signal power level in the $i$ th eigenchannel. Therefore, the form in (7) explicitly shows the relationship between MIMO capacity and the model in (5), although here the channels may have unequal SNR values $\Lambda_{i i} / \sigma^{2}$.

1) Water-Filling Capacity: Determining capacity involves identifying the covariance $\mathbf{R}_{x}$ that maximizes (6). If the scheme presented in Section II-C based on the SVD of $\mathbf{H}$ is used, then we see that $\mathbf{x}^{\prime}=\mathbf{V}^{H} \mathbf{X}$ (since $\mathbf{V}$ is unitary) and define $\mathbf{R}_{x}^{\prime}=$ $\mathrm{E}\left\{\mathbf{x}^{\prime} \mathbf{x}^{\prime H}\right\}=\mathbf{V}^{H} \mathbf{R}_{x} \mathbf{V}$. With this definition, it can be shown that $\mathbf{R}_{x}^{\prime}$ must be diagonal to maximize (6)[4]. Note that this is consistent with the notion that the unencoded transmit streams represented in $\mathbf{x}^{\prime}$ are independent. The capacity expression then becomes

$$
C=\max _{\left\{\mathbf{R}_{x}^{\prime}: \sum_{i} R_{x, i i}^{\prime} \leq P_{T}\right\}} \sum_{i=1}^{N_{R}} \log _{2}\left(1+\frac{S_{i i}^{2} R_{x, i i}^{\prime}}{\sigma^{2}}\right)
$$

where $R_{x, i i}^{\prime}$ represents the optimal transmit power on the $i$ th unencoded stream ( $i$ th orthogonal eigenpattern), and $S_{i i}^{2}$ is the power gain of the $i$ th eigenchannel. The values of $R_{x, i i}^{\prime}$ that maximize (8) can be determined using Lagrange multipliers to obtain the water-filling solution [4], [11], [13], [14]. This method allocates power to the high-gain channels and in general does not use weaker channels.

A simple computational example serves to illustrate the capacity gains available from this solution. Fig. 4 shows the cumulative distribution function (CDF) of capacity obtained via the water-filling solution for the cases where $\left(M=N_{T}, N=\right.$ $\left.N_{R}\right)=(1,1),(4,4)$, and $(12,4)$ [8]. The channel matrix $\mathbf{H}$ is constructed using Monte Carlo simulations with the channel model discussed in Section III-A2. The SNR for the $(1,1)$ case is $20 \mathrm{~dB}$. Significant capacity gain is observed when moving from 1 to 4 antennas. For the case $(12,4)$, only four parallel channels can be constructed from the system. Therefore, the increased capacity stems from improving the quality of these channels (increasing the magnitudes of the four largest singular values of $\mathbf{H}$ ) due to enhanced ability to create transmit radiation patterns that best excite the propagation environment.

2) Uninformed Transmitter Capacity: When the transmitter does not know $\mathbf{H}$, it can equally divide the power among the transmit antennas to form $N_{T}$ independent streams, or $\mathbf{R}_{x}=\left(P_{T} / N_{T}\right) \mathbf{I}$. Substitution into (6) results in the uninformed transmit capacity [2]

$$
C_{U T}=\log _{2}\left|\mathbf{I}+\frac{P_{T}}{N_{T} \sigma^{2}} \mathbf{H} \mathbf{H}^{H}\right| .
$$

For full-rank channel matrices at high SNR, the penalty paid for an uninformed transmitter is relatively small [14].

3) Channel Matrix Normalization: Since capacity depends on receive SNR, it is important to properly normalize channel 


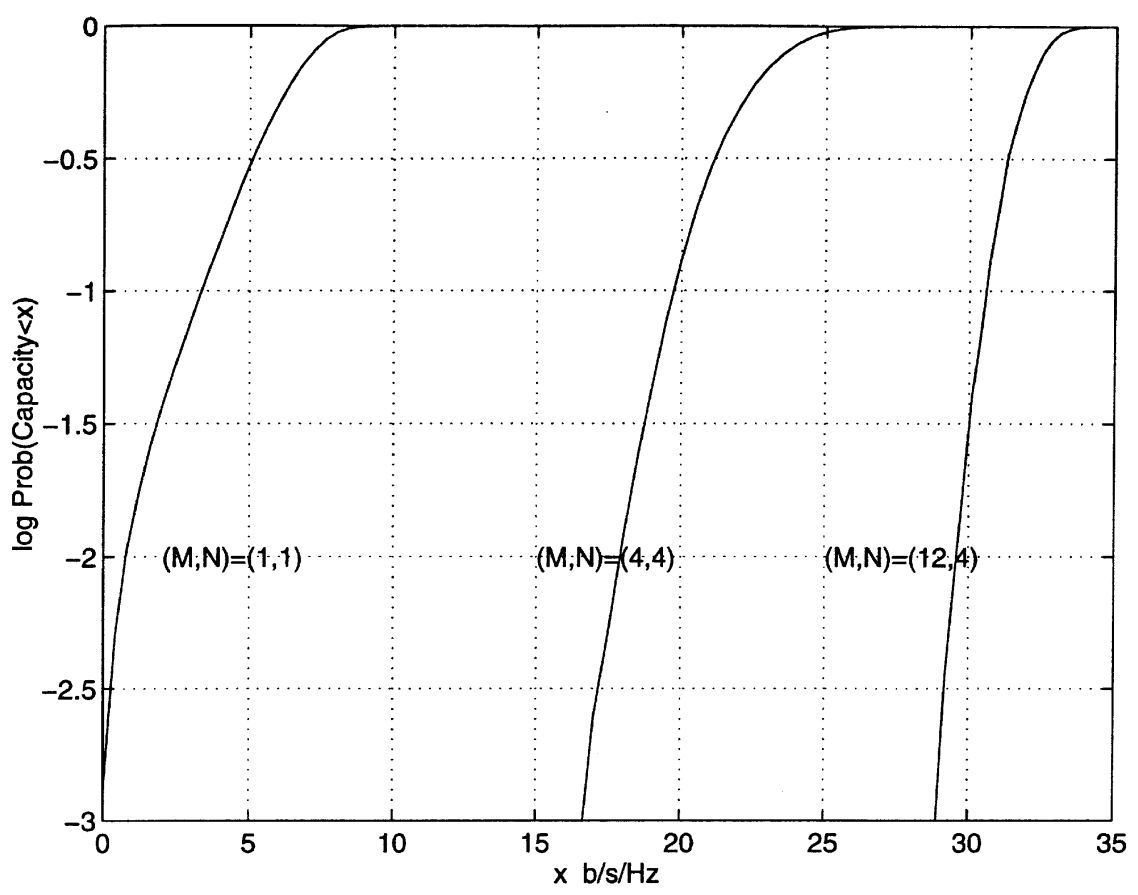

Fig. 4. CDF of capacity for $\left(M=N_{T}, N=N_{R}\right)=(1,1),(4,4)$, and $(12,4)$ for a simple channel model and a $(1,1)$ SNR of $20 \mathrm{~dB}$. Reprinted from [8] with permission (@ 2000 IEEE).

matrices for correct interpretation of results. For channel matrices $\mathbf{H}^{(n)}, 1 \leq n \leq N$, normalized channel matrices are computed as

$$
\mathbf{H}_{\text {norm }}^{(n)}=\mathbf{H}^{(n)}\left[\frac{1}{N N_{T} N_{R}} \sum_{n=1}^{N}\left\|\mathbf{H}^{(n)}\right\|_{F}^{2}\right]^{-1 / 2}
$$

where $\|\cdot\|_{F}$ is the Frobenius norm. When normalized matrices are used in the capacity expressions, $\rho=P_{T} / \sigma^{2}$ represents the average SNR of a single antenna system and is referred to as the single-input single-output (SISO) SNR. The differences in path loss among a number of channel matrices may be removed by normalizing each matrix independently, or $N=1$. Using $N>$ 1 preserves the relative power levels among the $N$ channels.

\section{E. Diversity and Spatial Multiplexing}

We have established that MIMO systems exploit the channel spatial degrees of freedom to increase communication performance. In traditional antenna diversity, these resources are used to transmit and/or receive duplicate copies of a single information stream to increase the reliability of detection. In contrast, spatial multiplexing indicates sending distinct information streams over the channels to increase throughput and spectral efficiency. The mix of diversity and spatial multiplexing accomplished with a MIMO system will depend on the throughput and quality-of-service requirements of the application [15].

This relationship implies that the traditional mechanisms used in diversity systems for reducing branch signal correlation generally work to improve MIMO performance as well. We emphasize, however, that low correlation is a necessary but not sufficient condition for good MIMO performance, since the propagation environment must also possess the appropriate characteristics (keyhole channels discussed in Section III-C support this statement). This low correlation is achieved when each antenna provides a unique weighting to each individual multipath component based on its DOD/DOA. This weighting can be on the arrival phase due to antenna location (spatial diversity), or on magnitude and phase due to antenna pattern (angle diversity) or polarization characteristics (polarization diversity) [5], [16]. Many systems use some combination of these mechanisms. Note that low correlation generally occurs for a large set of multipaths with large angular spread. The rich scattering required to achieve this condition generally also produces low SNR, which in turn decreases the channel capacity [17]-[19].

Channel measurements have been conducted to explore the effect of branch correlation on MIMO channel capacity. As an example, we examine data taken indoors at $2.45 \mathrm{GHz}$ using uniform linear arrays of quarter-wavelength dipoles [20]. The number of elements in the array are varied while the overall array length is maintained at 2.25 $\lambda$. Fig. 5 shows the resulting complementary CDF (CCDF) of capacity per number of transmit and receive antennas. Monte Carlo simulations using the channel model in Section III-A2, which neglects signal correlation, are also shown. The results indicate an excellent agreement between the measured and ideal $2 \times 2$ channel due to the wide antenna separation and resulting low correlation. As we pack more antennas into our array, the capacity per antenna drops due to higher correlation between adjacent elements. Several other studies have used experimental observations and analytical or simulation results to arrive at the same conclusion that increased signal correlation significantly degrades MIMO performance [21]-[26].

\section{MIMO CHANNEL MEASUREMENT AND MODELING}

Assessing the performance of MIMO systems in realistic environments requires a detailed description of the multipath 


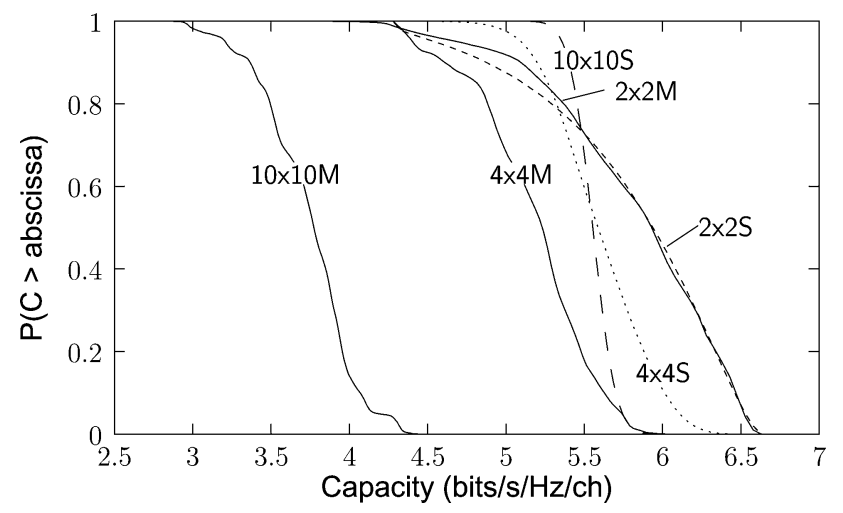

Fig. 5. Capacity CCDF's per number of antennas for transmit/receive arrays of increasing number of elements. Suffixes $\mathrm{M}$ and $\mathrm{S}$ correspond to measured (solid lines) and simulated (dashed lines) channels, respectively. The array length is $2.25 \lambda$ for all cases.

channel. This description must go beyond traditional models or measurement campaigns, as we must accurately represent a matrix of transfer functions. In some cases, channel measurements are used to fully characterize these channels. However, since relatively few such campaigns have been performed and the resulting data is not widely available, many researchers have turned to channel models that capture the key behaviors observed in the experimental data [27]-[31]. When accurate, these models facilitate performance assessment of potential space-time coding approaches in realistic propagation environments.

\section{A. Transfer Matrix Characterization}

1) Transfer Matrix Measurement: The most straightforward approach to characterizing the MIMO wireless channel is to deploy a system that directly measures the $N_{R} \times N_{T}$ channel matrix $\mathbf{H}$. In this case, all components in the top box in Fig. 2 are embedded in the channel, and the measurements will only be applicable for the analysis of systems employing the same array configurations and antenna elements.

A variety of measurements have appeared using such direct measurement platforms [17], [20], [32]-[55]. Results from such campaigns include channel capacity, signal correlation structure (in space, frequency, and time), channel matrix rank, path loss, delay spread, and a host of other quantities. Many of the deployed instruments use a true array system, where all antennas operate simultaneously. Such systems most closely model realworld MIMO communication and can accommodate channels that vary in time. The drawbacks, however, include the mutual coupling of the antenna array and the cost of the parallel transmit and receive electronics. The measured data shown in Fig. 5 was obtained with such a system.

Fig. 6 shows the evolution of capacity with distance traveled for an urban environment (Manhattan, New York City) obtained with a true array system at a SNR of $10 \mathrm{~dB}$ [37]. The linear transmit and rectangular receive arrays each used 16 elements of alternating polarization. This data shows the variation in capacity as the quality of the channel varies with position. More will be addressed about the "predicted" curve in this plot in Section III-A2.

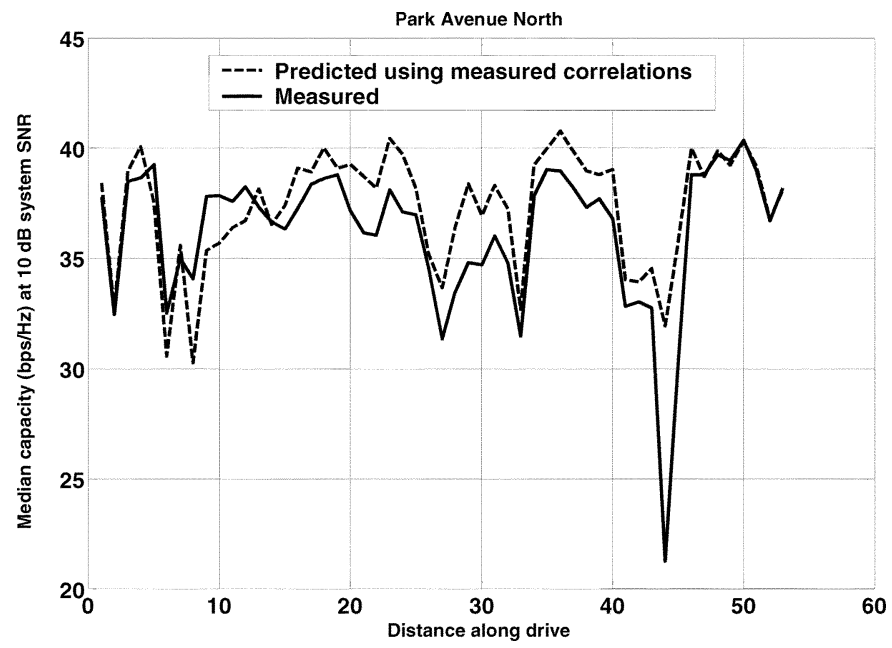

Fig. 6. Measured capacity as a function of location using 16-element transmit/receive arrays in an urban environment compared with simulated capacities. Reprinted from [37] with permission (C 2003 IEEE).

Other measurement systems employ either switched array or virtual array architectures. Switched array designs use a single transmitter and single receiver to measure the transfer function, with high-speed switches sequentially connecting all array elements to the electronics [56], [57]. Switching times for such systems are generally very low ( $2 \mu$ s to $100 \mathrm{~ms})$, indicating that the measurement over all antenna pairs can be conducted before the channel changes appreciably for most environments of practical interest. Virtual array instruments use precision displacement (or rotation) of a single antenna element to prescribed locations [18], [58], [59]. Although this method has the advantage of eliminating mutual coupling, a complete channel matrix measurement often takes several seconds or minutes, requiring a long channel mean stationary time. Therefore, virtual arrays are most suitable for fixed indoor measurement campaigns when activity is low.

2) Transfer Matrix Modeling: The simplest channel models directly compute the transfer matrix $\mathbf{H}$ based upon a statistical description. For example, in a non line-of-sight (NLOS) propagation environment, it is commonly assumed that the transfer function between one transmit and one receive antenna will have a magnitude and phase that follow Rayleigh and uniform distributions, respectively [5]. This combination indicates that the individual complex elements of $\mathbf{H}$ are circularly symmetric complex Gaussian random variables. In this case, the distribution is completely specified by the complex covariance matrix $\mathbf{R}=\mathrm{E}\left\{\mathbf{h h}^{H}\right\}$, where $\mathbf{h}=\operatorname{Vec}(\mathbf{H})$ and $\operatorname{Vec}(\cdot)$ stacks the columns of the matrix argument into a single column vector. In cases where only power correlation information is available, the elementwise square root of the power covariance matrix (the covariance of the vector $\mathbf{p}$ with elements $\left|h_{i}\right|^{2}$ ) is sometimes used instead of the complex covariance matrix.

Many studies assume no correlation between the signals on different antennas, or $\mathbf{R}=\mathbf{I}$, leading to independent matrix entries. If correlation structure is to be included, a covariance matrix must be constructed either directly from measured data or from a correlation model. If the fading statistics at transmit 
and receive are assumed to be independent, a separable covariance structure, referred to as the "Kronecker" product model [37], [60]-[62], can be created with the form $\mathbf{R}=\mathbf{R}_{T} \otimes \mathbf{R}_{R}$, where $\mathbf{R}_{T}$ and $\mathbf{R}_{R}$ are covariance matrices for signals on the transmit and receive arrays, respectively, and $\otimes$ represents the Kronecker product. Furthermore, if the multipath arrivals are uniformly distributed in angle in the azimuthal plane, then these covariance matrices can be computed from [5]

$$
R_{\{T, R\}, i j}=J_{0}\left(\frac{2 \pi}{\lambda}\left\|\mathbf{r}_{i}-\mathbf{r}_{j}\right\|\right)
$$

where $J_{0}(\cdot)$ is the zeroth order Bessel function, $\mathbf{r}_{i}$ is the coordinate of the $i$ th antenna element in the $x y$ plane, and $\|\cdot\|$ is the vector norm.

This approach is very simple to implement, and therefore facilitates assessment of space-time codes using Monte Carlo simulation approaches. Some studies have shown that this model is highly effective in matching measured results for systems with up to four antenna elements [61], [62]. However, recent work has demonstrated key deficiencies in this Kronecker product model [63]. In fact, one study has demonstrated that the Kronecker structure leads to high errors not only in the computed capacity but also in the covariance matrix representation [64]. Fig. 7 shows a plot of the normalized error $\kappa=\| \hat{\mathbf{R}}-\hat{\mathbf{R}}_{T} \otimes$ $\hat{\mathbf{R}}_{R}\left\|_{F} /\right\| \hat{\mathbf{R}} \|_{F}$ as a function of $N_{R}=N_{T}$, where $\hat{\mathbf{R}}, \hat{\mathbf{R}}_{T}$, and $\hat{\mathbf{R}}_{R}$ represent the total, transmit, and receive covariance matrices, respectively, estimated from measured data. These estimates are computed from data taken over a measurement distance of about $4000 \lambda$. The two curves are for taking the expectation over the entire distance and over segments of lengths $400 \lambda$ (and then averaging the 10 covariance results). Very large error is apparent, particularly as the array sizes grow. The error is reduced when the computation is performed over larger segments, perhaps due to more variation in the structure of the propagation environment [64].

The "predicted" curve in Fig. 6 is obtained using this complex Gaussian model, but using covariance matrices estimated directly from the experimental data. While the general trend is accurate, clearly some discrepancies exist. Another study has confirmed this error in the capacity as well as error in the joint statistics of the resulting transfer matrix [65]. These results imply that while the statistics of the transfer matrix elements are marginally complex Gaussian, the joint statistics are not. Nevertheless, the simplicity of this model makes it an attractive starting point in the analysis of any MIMO system, and recent augmentations have been shown to provide some increased accuracy [66]. Improved accuracy can then be achieved using more sophisticated models as outlined below. An important research activity involves finding models with this level of simplicity that properly capture the channel behavior.

\section{B. Multipath Characterization}

Another philosophy regarding MIMO channel characterization is to directly describe the properties of the physical multipath propagation channel, represented by the small dotted box in Fig. 2. Ideally, such measurements are independent of the properties associated with the measurement antennas. While early

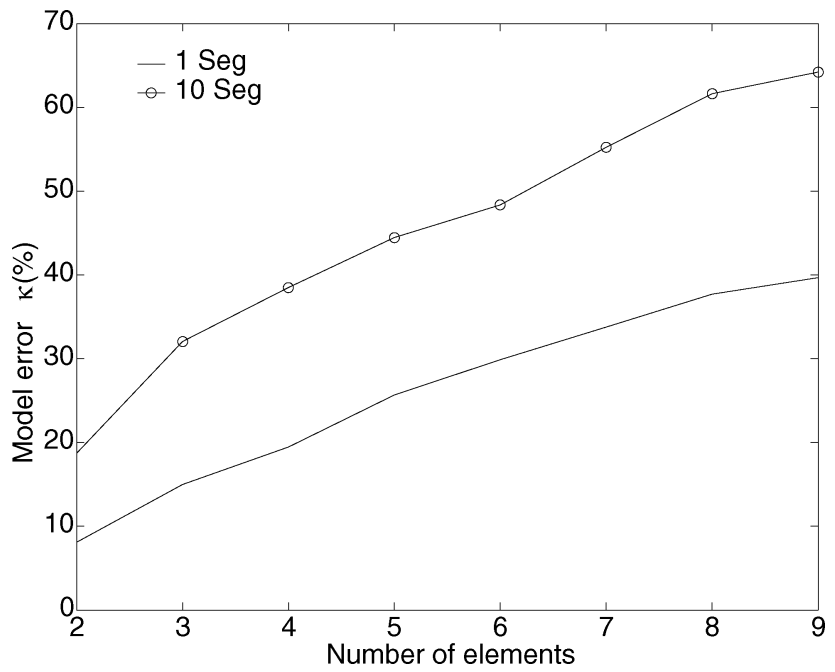

Fig. 7. Error associated with estimating the covariance matrix of measured data using the Kronecker product structure as a function of the array sizes.

work in this field has set the stage for more current activities, it is the recent double-directional channel characterization that is most useful for MIMO channel assessment [67]. This representation models the channel in wavenumber space according to the directional impulse response $M\left(\mathbf{k}, \mathbf{k}^{\prime}, t\right)$, where $\mathbf{k}$ and $\mathbf{k}^{\prime}$ are vectors associated with receive and transmit wavenumber (direction), $t$ is time, and single polarization has been assumed. Assuming only far-field propagation mechanisms, $\mathbf{k}$ and $\mathbf{k}^{\prime}$ are unit vectors in the propagation direction, with wave propagation at the speed of light. For simplicity, the fields at the receiver are typically modeled as a discrete set of plane-waves according to

$$
M\left(\mathbf{k}, \mathbf{k}^{\prime}, t\right)=\sum_{\ell=1}^{L} \beta_{\ell} e^{j \phi_{\ell}} \delta\left(t-\tau_{\ell}\right) \delta\left(\mathbf{k}-\mathbf{k}_{\ell}\right) \delta\left(\mathbf{k}^{\prime}-\mathbf{k}_{\ell}^{\prime}\right)
$$

where $\delta(\cdot)$ is the Dirac delta function, and the $\ell$ th multipath component has amplitude $\beta_{\ell}$, phase $\phi_{\ell}$, time of arrival (TOA) $\tau_{\ell}$, direction of arrival (DOA) $\mathbf{k}_{\ell}$, and direction of departure (DOD) $\mathbf{k}_{\ell}^{\prime}$. Intuitively, each multipath component corresponds to a plane wave arriving at a specific time and direction at the receiver due to energy launched in a specific transmit direction. When the true channel behavior is well represented by (12), the multipath parameters may be measured using conventional beamforming [68], basis matching techniques [69], or parametric estimation algorithms such as ESPRIT [67], [70], [71].

With the directional impulse response known, the transfer matrix elements for particular transmit and receive antenna arrays (assuming ideal voltage sampling) are obtained through the relationship shown in (13) at the bottom of the next page, where $g_{i}$ and $g_{m}^{\prime}$ are field radiation patterns and $\mathbf{r}_{i}$ and $\mathbf{r}_{m}^{\prime}$ are phase center coordinates for the $i$ th receive and $m$ th transmit antennas in the receive and transmit spaces, respectively. If $M\left(\mathbf{k}, \mathbf{k}^{\prime}, t\right)$ is specified as in (12), the elements of the wide-band channel matrix become

$$
\begin{aligned}
& H_{i m} \\
& \quad=\sum_{\ell=1}^{L} \beta_{\ell} g_{i}\left(\mathbf{k}_{\ell}\right) g_{m}^{\prime}\left(\mathbf{k}_{\ell}^{\prime}\right) \exp \left[j\left(-\omega \tau_{\ell}+\mathbf{k}_{\ell} \cdot \mathbf{r}_{i}+\mathbf{k}_{\ell}^{\prime} \cdot \mathbf{r}_{m}^{\prime}\right)\right] .
\end{aligned}
$$




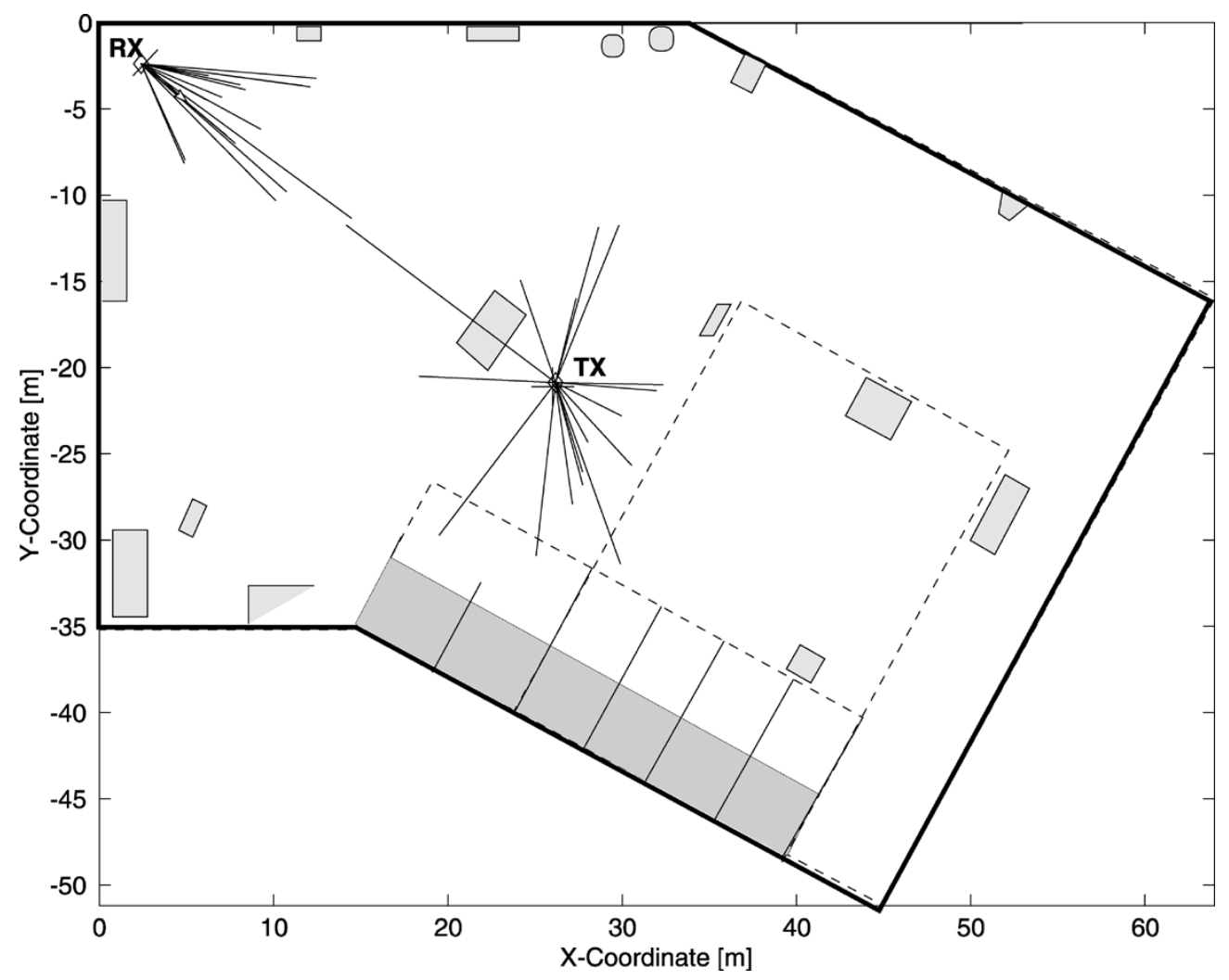

Fig. 8. Diagram of an outdoor backyard environment with plane wave departure and arrivals obtained through measurement. Reprinted from [72] with permission (C 2002 IEEE).

Fig. 8 shows an outdoor scene (backyard) with the estimated DOA/DOD of the multipaths obtained using ESPRIT on data taken with a virtual transmit array/switched receive array channel probing system [72]. The system center frequency and bandwidth were $5.2 \mathrm{GHz}$ and $120 \mathrm{MHz}$, respectively. The vector length corresponds to the gain of each estimated path. This data can then be used to assess the MIMO performance for any desired transmit/antenna configuration as well as for local movement of the arrays within this environment. Fig. 9 plots the $10 \%$ outage capacity (which simply means that the capacity is lower than this only $10 \%$ of the time), for different array sizes using this concept. This post-measurement flexibility is not possible with direct measurements of $\mathbf{H}$, since in that case the antennas become part of the measured channel.

A limitation of characterizing the measured channel as discrete plane waves is that realistic scattering often generates fields poorly represented by this model. For example, rough surfaces and random media produce diffuse scattering, generating a continuum of departure and arrival directions. Also, if scattering objects are not in the far field of the antennas, curved wavefronts or evanescent fields may be present that do not conform to the model. Finally, even if the multipath is discrete, the multipath components may be so numerous that the system resources (number of elements, bandwidth) are inadequate to resolve them all. When the assumptions of the

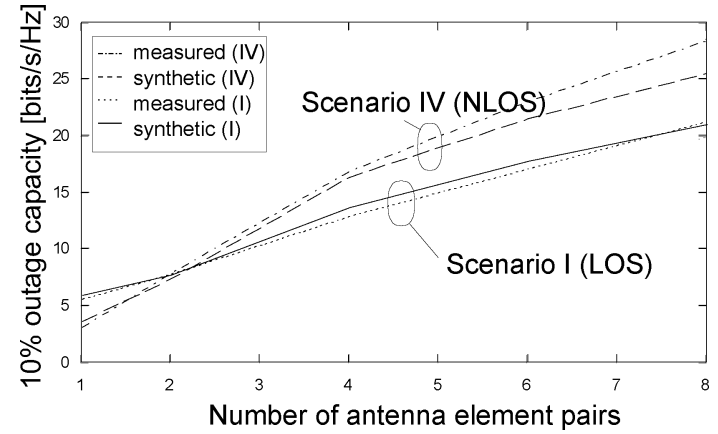

Fig. 9. Capacity versus array size obtained from simulations using measured multipath parameters in line-of-sight (LOS) and nonline-of-sight (NLOS) environments. Reprinted from [72] with permission (ㄷ 2002 IEEE).

model are violated, channels reconstructed from (12) will most likely under predict capacity due to lost information and power. More research is needed to determine the properties of realistic propagation and to evaluate the accuracy of channel response reconstructions based on (12).

Models capturing multipath behavior range in complexity from deterministic site-specific ray-tracing to simpler statistical descriptions. A fairly complete review of directional modeling techniques can be found in [27]. Here we briefly describe a few techniques that have recently been applied to MIMO modeling.

$$
H_{i m}=\iiint M\left(\mathbf{k}, \mathbf{k}^{\prime}, t\right) g_{i}(\mathbf{k}) g_{m}^{\prime}\left(\mathbf{k}^{\prime}\right) \exp \left[j\left(-\omega t+\mathbf{k} \cdot \mathbf{r}_{i}+\mathbf{k}^{\prime} \cdot \mathbf{r}_{m}^{\prime}\right)\right] d \mathbf{k} d \mathbf{k}^{\prime} d t
$$


Further work is needed to explore these models and improve their ability to represent key physical channel characteristics.

1) Deterministic Ray-Tracing: Deterministic site-specific modeling begins by creating a two dimensional (2-D) or 3-D computer model of a propagation environment. The response of the model to electromagnetic excitation may then be computed through computational techniques. Such models can also provide statistical channel information by applying Monte Carlo analysis on many random transmit/receive locations and/or model geometries.

ffRay-tracing [73]-[78] has emerged as the most popular technique for the analysis of site-specific scenarios, due to its ability to analyze very large structures with reasonable computational resources. The technique is based on geometrical optics, often supplemented by diffraction theory to enhance accuracy in shadowed regions. Recent studies have further combined ray-tracing with full-wave electromagnetic solvers to model objects with features that are comparable to the illumination wavelength [79], [80].

Ray-tracing techniques have demonstrated reasonable accuracy in predicting large-scale path loss variation, with error standard deviations of 3-7 dB being reported. However, preliminary comparisons of ray-tracing predictions with measurements indicate that the simulations tend to underestimate MIMO channel capacity [81], likely due more to over-simplification of the geometrical scenario representation than failure of the electromagnetic simulation approach. Other recent work [82] shows promising agreement in exact DOAs of measured and simulated micro-cells. In this case, the results can be combined with a random distribution for phase [82]-[85] to create a complete model. Further work is needed to identify how much model detail is required to correctly represent the channel.

Ray-tracing simulations have been used to study MIMO channel characteristics such as spatial-signature variation with small-scale movement [86], capacity variation with array location and antenna spacing [26], [87], and angular clustering of multipath arrivals [88]. Ray-tracing studies have also led to the development of simpler statistical models such as those described in Section III-B.3.

2) Geometric Discrete Scattering Models: Due to the high computational cost of rigorous ray-tracing simulations, more approximate models have appeared that assume simplified geometries and scattering mechanisms. For example, in [89]-[96], scatterers are modeled as discrete objects located about the receiver and/or transmitter. These objects can represent site-specific obstacles, or their locations and cross-sections can be defined statistically. Assuming either a single-bounce or double-bounce scattering mechanism, the channel response may be rapidly computed. These models also allow for dynamic channel evolution by computing the response as the transmitter or receiver moves through the environment. Finally, statistical scatterer characterization can lead to convenient, closed-form statistical distributions on delay spread, angular spread, and spatial correlation [97]-[100].

3) Statistical Cluster Models: Statistical cluster models directly specify distributions on the multipath DOD/DOA, TOA, and amplitude. Most current models are based on initial work by Turin, et al. [101] who observed that multipath components can be grouped into clusters that decay exponentially with in-

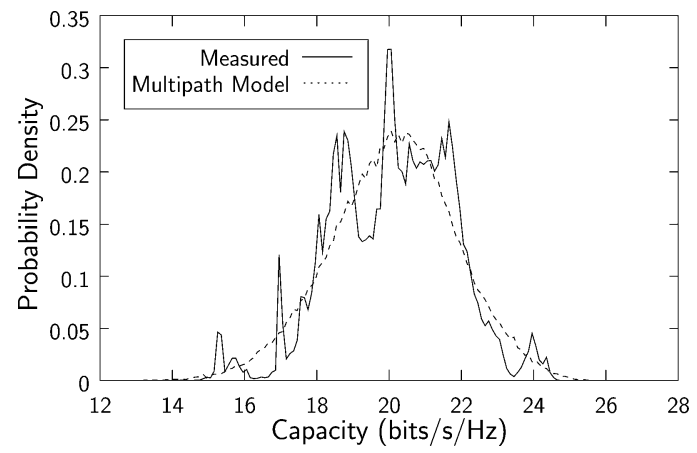

Fig. 10. Comparison of capacity PDFs for $4 \times 4$ measured data and multipath channel model simulations.

creasing delay time. Intuitively, a single cluster of arrivals might correspond to a single scattering object and the arrivals within the cluster arise due to smaller object features. Later work applied the model to indoor scenarios [102] and added directional information [65], [69], [93], [103]. In more advanced models, the "birth" and "death" of clusters due to movement of the subscriber can be taken into account [104]. Statistical descriptions of the multipath arrival parameters have been obtained through measurements [69], [92], [105]-[109] and ray-tracing [104]. Other work has combined the cluster and discrete scattering models to include both distant and local scattering [110], [111].

Provided that the underlying statistical distributions are properly specified, these models can offer highly accurate channel representations (in a statistical sense). Fig. 10 compares probability density functions (PDFs) of capacity obtained from measurements and Monte Carlo simulations $\left(10^{5}\right.$ channel realizations) from one such model for a $4 \times 4$ system operating in an indoor environment [65]. The good agreement observed between the measured and simulated results is superior to that obtained with direct transfer matrix modeling approaches for fixed covariance.

\section{Keyhole (Pinhole) Channels}

Keyhole channels occur when, despite the presence of scatterers local to the transmitting and receiving nodes, the multipaths travel from the area local to the transmitter to the area local to the receiver via one dominant path. A simple example of this would be two outdoor regions connected by a single tunnel. At the receiver, the locally scattered components, despite potentially large angle spread, will all contain essentially the same information, leading to little or no capacity gain. A variety of simple studies have demonstrated the possibility of such channels and have quantified the performance of MIMO systems in this environment [30], [112]-[114]. These findings underscore the fact that MIMO system performance cannot always be determined by traditional average metrics such as antenna signal correlation, since channel capacity depends on each instantaneous realization of the channel matrix and not just average correlation properties. We point out that around " 43 " on the horizontal axis in Fig. 6, we observe a large measured capacity drop while the simulated capacity remains relatively high (implying low correlation). This point corresponds to measurement through a tunnel and appears to be a result of keyhole propagation. 


\section{THE IMPACT OF ANTENNAS ON MIMO PERFoRMANCE}

As previously mentioned, MIMO systems perform best when the transfer matrix is full rank, a situation achieved when the correlation between signals on the different antennas is low. This section discusses how antenna properties such as pattern, polarization, array configuration, and mutual coupling can impact this correlation.

\section{A. Element Radiation Pattern}

Angle diversity results when antennas have distinct radiation patterns. Mathematically, assuming two antennas with the same phase center (or closely spaced phase centers), a single incident wave polarization, and that the arrival angles of the multipath components satisfy a PDF $f_{\Omega}(\Omega)$, we desire the signal correlation [115]

$$
\rho_{p}=\int f_{\Omega}(\Omega) e_{1}(\Omega) e_{2}^{*}(\Omega) d \Omega
$$

where $e_{i}(\Omega)$ is the pattern of the $i$ th antenna, to be small. When the antenna phase centers are closely spaced so that angle diversity is the dominant factor, the essentially omnidirectional pattern created by most small elements results in relatively large values of $\rho_{p}$, leading to low capacity. However, when element patterns are appropriately designed to minimize (15), then capacity gains are possible. One suggested approach for realizing such a situation involves the use of multi-mode antennas where the patterns for different modes exhibit high orthogonality (low correlation) in the form of (15)[116]. Finding other antenna topologies that offer this orthogonality in a compact form factor remains an area of active research.

Another important aspect of element radiation pattern involves the manner in which the antenna excites the multipath environment. As an example, a recent study has compared the capacity performance of dipole antennas with that of higher-gain spiral antennas whose radiation patterns tend to be more directive toward $45^{\circ}$ and $135^{\circ}$ in elevation [117]. Fig. 11 shows measured (using a switched array system) and simulated (using a statistical path-based channel model) capacities for the two antennas in an indoor environment. The results show that the lower gain dipoles offer superior capacity (by about 10\%), since these antennas put more energy into the horizontal plane where most of the multipath components are concentrated.

\section{B. Array Configuration}

It is important to emphasize that the transfer matrix $\mathbf{H}$ in (1) depends not only on the propagation environment but also on the array configurations. The question becomes which array topology is best in terms of maximizing capacity (perhaps in an average sense over a variety of propagation channels) or minimizing symbol error rates. This is difficult to answer definitively, since the optimal array shape depends on the site-specific propagation characteristics, although some general observations are possible.

First, there is a notable study where several different array types were explored for both the base station and the mobile unit in an outdoor environment [45]. The base station antennas included single and dual polarization array and multibeam

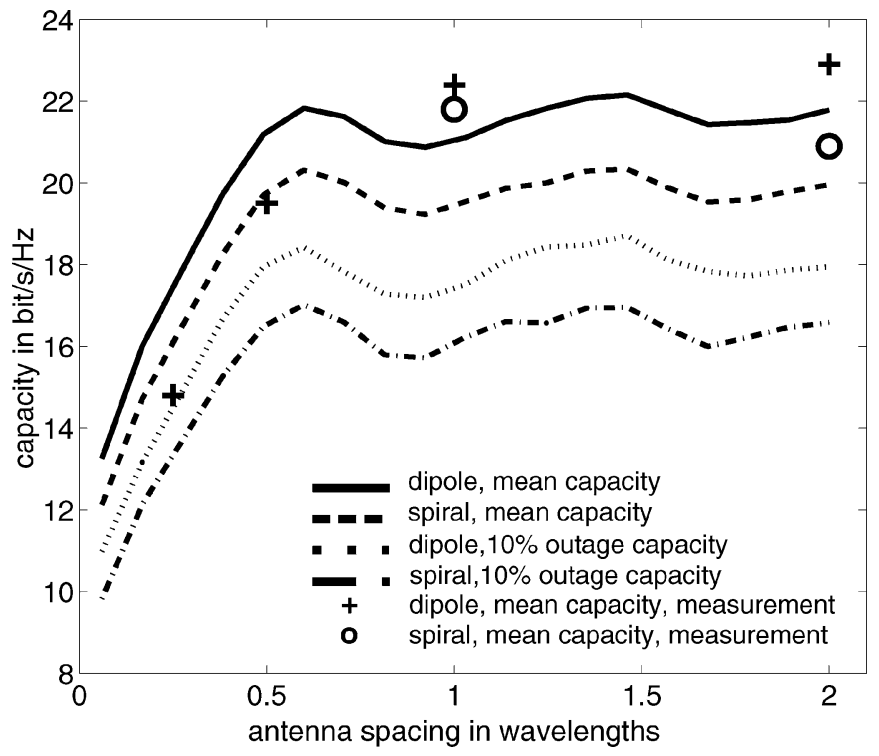

Fig. 11. Simulated and measured outage capacities for arrays of 2 dipole and spiral antennas in an indoor environment as a function of antenna spacing. Reprinted from [117] with permission (@ 2002 IEEE).

structures. The arrays on the mobile were constructed from monopoles to achieve spatial, angle, and/or polarization diversity. All of the array configurations provided very similar performance, with the exception of the multibeam base station antennas which resulted in a 40-50\% reduction in measured capacity since generally only one of the beams pointed in the direction of the mobile. These results suggest that average capacity is relatively insensitive to array configuration.

The conclusions drawn above are based on comparisons of average capacity for different array configurations. Alternatively, we can consider an adaptive system that selectively connects a subset of available antennas to the electronic modules. Studies have shown that an intelligently-selected sub-array can provide improved capacity [118]-[122] or lower probability of symbol error [123] relative to the performance of fixed arrays.

Finally, we observe that for single antenna systems, the capacity bound in (4) is independent of the antenna (other than the antenna gain). The strong dependence of MIMO capacity on array configuration is therefore troubling since this number is not a true upper bound on the physical channel throughput. In response to this, recent work has formulated the Intrinsic Capacity for a specific channel and spatial antenna apertures independent of the array configuration [124]. This creates new research avenues in identifying antenna elements and arrays that provide optimal or near-optimal performance.

\section{Element Polarization}

Recent work has suggested that in a rich multipath environment, sensing the three Cartesian vector components of the electric and magnetic fields can provide six uncorrelated signals at the receiver [125]. This conclusion can be understood by recognizing that for large multipath angle spread, the combined polarization and angle diversity offered by three orthogonally-oriented electric and magnetic Hertzian dipoles at a point can lead to six uncorrelated signals [126], [127]. 


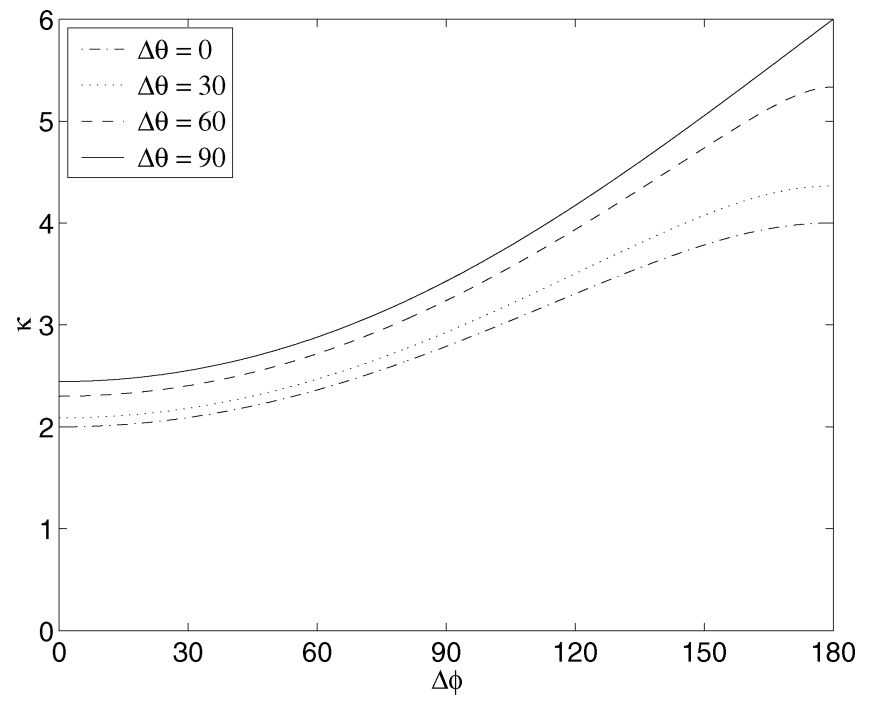

Fig. 12. Plot of the effective number of communication channels as a function of azimuthal angle spread for several different elevation angle spreads.

Fig. 12 plots the effective number of polarization-based communication channels as a function of multipath arrival spread in azimuth $(-\Delta \phi<\phi<\Delta \phi)$ for several different elevation spreads $(-\Delta \theta<\theta<\Delta \theta)$. For a single propagation path ( $\Delta \theta=\Delta \phi=0$ ), only two channels exist corresponding to the two possible polarizations of the incident plane wave. Six independent communication modes are enabled only for full multipath angle spread.

From a practical standpoint, constructing a multi-polarized antenna that can achieve this bound is problematic. Using halfwavelength dipoles and full-wavelength loops leads to strong mutual coupling and nonideal pattern characteristics that can reduce the number of independent channels. One interesting geometry is a cube consisting of dipole antennas to obtain a high-degree of polarization diversity in a compact form [128]. The design and fabrication of other practical antennas that can exploit polarization remains an area where further research is warranted.

Finally, in the common case where two polarizations are used, typical scattering leads to a co-polarized received signal that is 4 to $10 \mathrm{~dB}$ higher than the cross-polarized signal level [129]. The result is a transfer matrix that exhibits low correlation (high diversity) coupled with weak channel gain between the two orthogonally polarized channels (reduced SNR) [16], [20], [130]-[133]. Experimental results demonstrate capacity gains of around 10-20\% from using dual-polarization over single-polarization spatially-separated elements in an indoor environment [20]. The key advantage to using two polarizations is that regardless of the environment, at least two parallel channels are enabled.

\section{Mutual Coupling}

Antenna mutual coupling is a key issue of concern for MIMO systems [134], [135]. It has long been known that the pattern distortion from close antenna spacing creates angle diversity that can lead to reduced signal correlation [115], [136]-[142]. More comprehensive studies have examined the effect of coupling and antenna termination on the capacity [143]-[146]. These

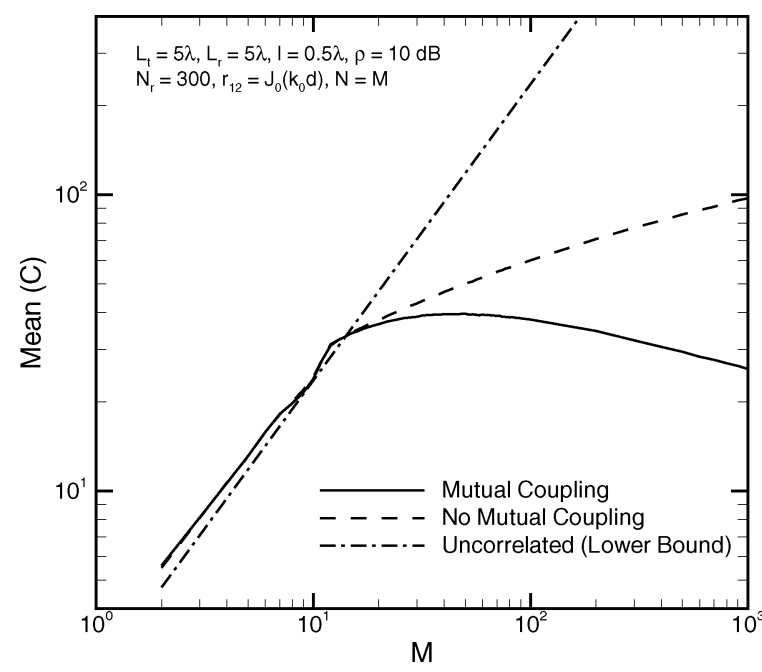

Fig. 13. Impact of mutual coupling on arrays of length $5 \lambda$ as the number of elements increases. Reprinted from [146] with permission (C) 2002 IEEE).

approaches construct the transfer function relating the signals at the receiver electronics to signals input at the transmit antenna terminals and accurately compute the received SNR on each branch. Two main conclusions have surfaced as a result of these studies. First, because of the induced angle diversity combined with improved power collection capability of coupled antennas [134], the capacity of two coupled dipoles can be higher than that of uncoupled antennas (through proper termination), particularly for small dipole spacing where coupling is high [143], [144]. Second, for a fixed-length array, the strong coupling between elements packed into the same physical space will ultimately lead to an upper bound on capacity performance. For example, Fig. 13 plots the capacity as a function of the number of transmit and receive half-wavelength dipoles $\left(N_{T}=N_{R}=M\right)$ packed into a linear array of length $5 \lambda[146]$. The propagation (field) channel model is that in Section III-A2 with covariance computed as the product of Bessel functions [similar to that in (11)]. This model is then augmented to include the mutual impedance of the antennas and terminations using network theory. Three hundred Monte Carlo realizations are used to obtain the mean capacities given for the case where the antennas are terminated in a self-impedance match. As can be seen, this analysis predicts significant capacity reduction compared to a system where coupling is neglected for spacing smaller than about $\lambda / 2$, leading to an upper capacity bound for a given aperture length.

\section{CONCLUSION}

This paper has provided a tutorial on the operation of MIMO wireless communication systems and illustrated how multiple antennas can lead to increased system capacity for multipath communication channels. It has also offered a review of recent research activities and findings related to antennas and propagation in MIMO communications, demonstrating the large variety and volume of work that has recently been accomplished in this arena. This review has shown that issues related to antennas and electromagnetic propagation play a significant role in determining MIMO system performance. Furthermore, 
the paper has highlighted potential future research directions within this general field, revealing that a number of challenging problems remain unsolved. It will take continued collaborative efforts from researchers in electromagnetics, signal processing, and communication theory to ultimately exploit the potential of MIMO technology through practical implementation.

\section{REFERENCES}

[1] J. H. Winters, "On the capacity of radio communication systems with diversity in a Rayleigh fading environment," IEEE J. Select Areas Commun., vol. SAC-5, pp. 871-878, June 1987.

[2] G. J. Foschini and M. J. Gans, "On limits of wireless communications in a fading environment when using multiple antennas," Wireless Personal Commun., vol. 6, pp. 311-335, Mar. 1998.

[3] T. L. Marzetta and B. M. Hochwald, "Capacity of a mobile multiple-antenna communication link in Rayleigh flat fading," IEEE Trans. Inform. Theory, vol. 45, pp. 139-157, Jan. 1999.

[4] G. G. Raleigh and J. M. Cioffi, "Spatio-temporal coding for wireless communication,” IEEE Trans. Commun., vol. 46, pp. 357-366, Mar. 1998.

[5] W. C. Jakes, Microwave Mobile Communications. New York: IEEE Press, 1993.

[6] J. B. Andersen, "Role of antennas and propagation for the wireless systems beyond 2000," Wireless Pers. Commun., vol. 17, pp. 303-310, Jun. 2001.

[7] J. B. Andersen and R. G. Vaughan, "Transmitting, receiving, and scattering properties of antennas," IEEE Antennas Propagat. Mag., vol. 45, Aug. 2003

[8] J. B. Andersen, "Array gain and capacity for known random channels with multiple element arrays at both ends," IEEE J. Select Areas Commun., vol. 18, pp. 2172-2178, Nov. 2000.

[9] G. D. Golden, C. J. Foschini, R. A. Valenzuela, and P. W. Wolniansky, "Detection algorithm and initial laboratory results using V-BLAST space-time communication architecture," Electron. Lett., vol. 35, pp. 14-16, Jan. 1999.

[10] P. W. Wolniansky, G. J. Foschini, G. D. Golden, and R. A. Valenzuela, "V-BLAST: An architecture for realizing very high data rates over the rich-scattering wireless channel," in Proc. URSI ISSSE'98, Pisa, Italy, Sept.-Oct. 29-2, 1998, pp. 295-300.

[11] T. M. Cover and J. A. Thomas, Elements of Information Theory: John Wiley \& Sons, 1991

[12] D. Gesbert, M. Shafi, D. s. Shiu, P. J. Smith, and A. Naguib, "From theory to practice: An overview of MIMO space-time coded wireless systems," IEEE J. Select Areas Commun., vol. 21, pp. 281-302, Apr. 2003.

[13] T. K. Moon and W. C. Stirling, Mathematical Methods and Algorithms for Signal Processing: Prentice Hall, 2000.

[14] M. A. Khalighi, J. Brossier, G. Jourdain, and K. Raoof, "Water filling capacity of Rayleigh MIMO channels," in Proc. IEEE 12th Int. Symp. on Personal, Indoor and Mobile Radio Comm., vol. 1, San Diego, CA, Sept.-Oct. 30-3, 2001, pp. 155-158.

[15] L. Zheng and D. Tse, "Diversity and multiplexing: A fundamental tradeoff in multiple antenna channels," IEEE Trans. Inf. Theory, vol. 49, pp. 1073-1096, May 2003.

[16] J. P. Kermoal, L. Schumacher, F. Frederiksen, and P. E. Mogensen, "Polarization diversity in MIMO radio channels: Experimental validation of a stochastic model and performance assessment," in Proc. IEEE 54th Veh. Technol. Conf., vol. 1, Atlantic City, NJ, Oct. 7-11, 2001, pp. 22-26.

[17] D. P. McNamara, M. A. Beach, P. N. Fletcher, and P. Karlsson, "Capacity variation of indoor multiple-input multiple-output channels," Electron. Lett., vol. 36, pp. 2037-2038, Nov. 2000.

[18] M. Herdin, H. Ozcelik, H. Hofstetter, and E. Bonek, "Variation of measured indoor MIMO capacity with receive direction and position at 5.2 GHz," Electronics Letters, vol. 38, pp. 1283-1285, Oct. 2002.

[19] T. Svantesson and J. W. Wallace, "On signal strength and multipath richness in multi-input multi-output systems," in Proc. IEEE Int. Conf. Commun., vol. 4, Anchorage, AK, May 11-15, 2003, pp. 2683-2687.

[20] J. W. Wallace, M. A. Jensen, A. L. Swindlehurst, and B. D. Jeffs, "Experimental characterization of the MIMO wireless channel: Data acquisition and analysis," IEEE Trans. Wireless Commun., vol. 2, pp. 335-343, Mar. 2003.

[21] D. Shiu, G. J. Foschini, M. J. Gans, and J. M. Kahn, "Fading correlation and its effect on the capacity of multielement antenna systems," IEEE Trans. Commun., vol. 48, pp. 502-513, Mar. 2000.
[22] D. Chizhik, F. Rashid-Farrokhi, J. Ling, and A. Lozano, "Effect of antenna separation on the capacity of BLAST in correlated channels," IEEE Commun. Lett., vol. 4, pp. 337-339, Nov. 2000.

[23] N. Chiurtu, B. Rimoldi, and E. Telatar, "Dense multiple antenna systems," in Proc. IEEE Information Theory Workshop, Cairns, Australia, Sep. 2-7, 2001, pp. 108-109.

[24] P. Kyritsi, D. C. Cox, R. A. Valenzuela, and P. W. Wolniansky, "Correlation analysis based on MIMO channel measurements in an indoor environment," IEEE J. Select Areas Commun., vol. 21, pp. 713-720, Jun. 2003.

[25] S.-Q. Wei, D. Goeckel, and R. Janaswamy, "On the asymptotic capacity of MIMO systems with fixed length linear antenna arrays," in Proc. IEEE Int. Conf. Commun., vol. 4, Anchorage, AK, May 11-15, 2003, pp. 2633-2637.

[26] C.-N. Chuah, D. N. C. Tse, J. M. Kahn, and R. A. Valenzuela, "Capacity scaling in MIMO wireless systems under correlated fading," IEEE Trans. Inf. Theory, vol. 48, pp. 637-650, Mar. 2002.

[27] R. B. Ertel, P. Cardieri, K. W. Sowerby, T. S. Rappaport, and J. H. Reed, "Overview of spatial channel models for antenna array communication systems," IEEE Pers. Commun., pp. 10-21, Feb. 1998.

[28] K. I. Pedersen, J. B. Andersen, J. P. Kermoal, and P. Mogensen, "A stochastic multiple-input-multiple-output radio channel model for evaluation of space-time coding algorithms," in Proc. IEEE 52nd Veh. Technol. Conf., vol. 2, Boston, MA, Sept. 24-28, 2000, pp. 893-897.

[29] A. M. Sayeed, "Modeling and capacity of realistic spatial MIMO channels," in Proc. IEEE Int. Conf. Acoustics, Speach, and Signal Processing, vol. 4, Salt Lake City, UT, May 7-11, 2001, pp. 2489-2492.

[30] D. Gesbert, H. Bolcskei, D. A. Gore, and A. J. Paulraj, "Outdoor MIMO wireless channels: Models and performance prediction," IEEE Trans. Commun., vol. 50, pp. 1926-1934, Dec. 2002.

[31] C. Xiao, J. Wu, S.-Y. Leong, Y. R. Zheng, and K. B. Letaief, "A discrete-time model for spatio-temporally correlated mimo wssus multipath channels," in Proc. IEEE Wireless Comm. and Networking Conf., vol. 1, New Orleans, LA, Mar. 16-20, 2003, pp. 354-358.

[32] J. W. Wallace and M. A. Jensen, "Statistical characteristics of measured MIMO wireless channel data and comparison to conventional models," in Proc. IEEE 54th Veh. Technol. Conf., vol. 2, Atlantic City, NJ, Oct. 7-11, 2001, pp. 1078-1082.

[33] — , "Measured characteristics of the MIMO wireless channel," in Proc. IEEE 54th Veh. Technol. Conf., vol. 4, Atlantic City, NJ, Oct. 7-11, 2001, pp. 2038-2042.

[34] _ - "Experimental characterization of the MIMO wireless channel," in Proc. IEEE Antennas and Propagation Society Int. Symp., vol. 3, Boston, MA, July 8-13, 2001, pp. 92-95.

[35] - "Characteristics of measured $4 \times 4$ and $10 \times 10$ MIMO wireless channel data at 2.4-GHz," in Proc. IEEE Antennas and Propagation Society Int. Symp., vol. 3, Boston, MA, July 8-13, 2001, pp. 96-99.

[36] M. Lienard, P. Degauque, J. Baudet, and D. Degardin, "Investigation on MIMO channels in subway tunnels," IEEE J. Select Areas Commun., vol. 21, pp. 332-339, Apr. 2003.

[37] D. Chizhik, J. Ling, P. W. Wolniansky, R. A. Valenzuela, N. Costa, and K. Huber, "Multiple-input-multiple-output measurements and modeling in Manhattan," IEEE J. Select Areas Commun., vol. 21, pp. 321-331, Apr. 2003.

[38] P. Papazian, M. Gans, Y. Lo, and R. Dalke, "Capacity measurements for a $16 \times 16$ element BLAST array over a conducting ground plane," in Proc. IEEE 56th Veh. Technol. Conf., vol. 1, Vancouver, BC, Sep. 24-28, 2002, pp. 634-638.

[39] H. Xu, M. Gans, D. Chizhik, J. Ling, P. Wolniansky, and R. Valenzuela, "Spatial and temporal variations of MIMO channels and impacts on capacity," in Proc. IEEE Int. Conf. Commun., vol. 1, New York, NY, Apr.-May 28-2, 2002, pp. 262-266.

[40] H. Xu, M. Gans, N. Amitay, R. A. Valenzuela, T. Sizer, R. Storz, D. Taylor, M. McDonald, and C. Tran, "MIMO channel capacity for fixed wireless: Measurements and models," in Proc. IEEE 54th Veh. Technol. Conf., vol. 2, Atlantic City, NJ, Oct. 7-11, 2001, pp. 1068-1072.

[41] M. J. Gans, N. Amitay, Y. S. Yeh, H. Xu, T. C. Damen, R. A. Valenzuela, T. Sizer, R. Storz, D. Taylor, W. M. McDonald, C. Tran, and A. Adamiecki, "Outdoor BLAST measurement system at $2.44 \mathrm{GHz}$ : Calibration and initial results," IEEE J. Select Areas Commun., vol. 20, pp. 570-583, Apr. 2002.

[42] C. C. Martin, J. H. Winters, H. H. Zeng, N. R. Sollenberger, and A. Dixit, "Multiple-input multiple-output (MIMO) radio channel measurements and experimental implementation for EDGE," in Proc. 34th Asilomar Conf. Signals, Systems and Computers, vol. 1, Pacific Grove, CA, Oct.Nov. $29-1,2000$, pp. $738-742$. 
[43] C. C. Martin, J. H. Winters, and N. R. Sollenberger, "Multiple-input multiple-output (MIMO) radio channel measurements," in IEEE Sensor Array and Multichannel Signal Processing Workshop, Cambridge, MA, Mar. 16-17, 2000, pp. 45-46.

[44] — "Multiple-input multiple-output (MIMO) radio channel measurements," in Proc. IEEE Antennas and Propagation Society Int. Symp., vol. 1, Boston, MA, July 8-13, 2001, pp. 418-421.

[45] - "MIMO radio channel measurements: Performance comparison of antenna configurations," in Proc. IEEE 54th Veh. Technol. Conf., vol. 2, Atlantic City, NJ, Oct. 7-11, 2001, pp. 1225-1229.

[46] K. Yu, M. Bengtsson, B. Ottersten, D. McNamara, P. Karlsson, and M. Beach, "Second order statistics of NLOS indoor MIMO channels based on $5.2 \mathrm{GHz}$ measurements," in Proc. IEEE Global Telecomm. Conf., vol. 1, San Antonio, TX, Nov. 25-29, 2001, pp. 156-160.

[47] R. Stridh, B. Ottersten, and P. Karlsson, "MIMO channel capacity of a measured indoor radio channel at $5.8 \mathrm{GHz}$," in Proc. 34th Asilomar Conf. Signals, Systems and Computers, vol. 1, Pacific Grove, CA, Nov. 1, 2000, pp. 738-742.

[48] R. Stridh and B. Ottersten, "Spatial characterization of indoor radio channel measurements at $5 \mathrm{GHz}$," in IEEE Sensor Array and Multichannel Signal Processing Workshop, Cambridge, MA, Mar. 16-17, 2000, pp. 58-62.

[49] J. Medbo and J.-E. Berg, "Simple and accurate path loss modeling at $5 \mathrm{GHz}$ in indoor environments with corridors," in Proc. IEEE 52nd Veh. Technol. Conf., vol. 1, Boston, MA, Sept. 24-28, 2000, pp. 30-36.

[50] D. P. McNamara, M. A. Beach, and P. N. Fletcher, "Experimental investigation of the temporal variation of MIMO channels," in Proc. IEEE 54th Veh. Technol. Conf., Atlantic City, NJ, Oct. 7-11, 2001, pp. 1063-1067.

[51] —, "Spatial correlation in indoor MIMO channels," in Proc. IEEE 13th Int. Symp. on Personal, Indoor and Mobile Radio Comm., vol. 1, Lisboa, Portugal, Sep. 15-18, 2002, pp. 290-294.

[52] D. S. Baum, D. Gore, R. Nabar, S. Panchanathan, K. V. S. Hari, V. Erceg, and A. J. Paulraj, "Measurement and characterization of broadband MIMO fixed wireless channels at $2.5 \mathrm{GHz}$," in IEEE Int. Conf. on Personal Wireless Communications, Hyderabad, India, Dec. 17-20, 2000, pp. 203-206.

[53] V. Erceg, P. Soma, D. S. Baum, and A. J. Paulraj, "Capacity obtained from multiple-input multiple-output channel measurements in fixed wireless environments at $2.5 \mathrm{GHz}$," in Proc. IEEE Int. Conf. Commun., vol. 1, New York, NY, Apr.-May 28-2, 2002, pp. 396-400.

[54] M. D. Batariere, T. K. Blankenship, J. F. Kepler, T. P. Krauss, I. Lisica, S. Mukthavaram, J. W. Porter, T. A. Thomas, and F. W. Vook, "Wideband MIMO mobile impulse response measurements at $3.7 \mathrm{GHz}$," in Proc. IEEE 55th Veh. Technol. Conf., vol. 1, Birmingham, AL, May 6-9, 2002, pp. 26-30.

[55] J. P. Kermoal, L. Schumacher, P. E. Mogensen, and K. I. Pedersen, "Experimental investigation of correlation properties of MIMO radio channels for indoor picocell scenarios," in Proc. IEEE 52nd Veh. Technol. Conf., vol. 1, Boston, MA, Sept. 24-28, 2000, pp. 14-21.

[56] A. F. Molisch, M. Steinbauer, M. Toeltsch, E. Bonek, and R. S. Thoma, "Measurement of the capacity of MIMO systems in frequency-selective channels," in Proc. IEEE 53rd Veh. Technol. Conf., vol. 1, Rhodes, Greece, May 6-9, 2001, pp. 204-208.

[57] — "Capacity of MIMO systems based on measured wireless channels," IEEE J. Select Areas Commun., vol. 20, pp. 561-569, Apr. 2002.

[58] J. Medbo and J.-E. Berg, "Spatio-temporal channel characteristics at 5 GHz in a typical office environment," in Proc. IEEE 54th Veh. Technol. Conf., vol. 3, Atlantic City, NJ, Oct. 7-11, 2001, pp. 1256-1260.

[59] D. Kim, M. A. Ingram, and W. W. Smith, "Small-scale fading for an indoor wireless channel with modulated backscatter," in Proc. IEEE 54th Veh. Technol. Conf., Atlantic City, NJ, Oct. 7-11, 2001, pp. 1616-1620.

[60] S. L. Loyka, "Channel capacity of MIMO architecture using the exponential correlation matrix," IEEE Commun. Lett., vol. 5, pp. 369-371, Sept. 2001.

[61] J. P. Kermoal, L. Schumacher, K. I. Pedersen, P. E. Mogensen, and F. Frederiksen, "A stochastic MIMO radio channel model with experimental validation," IEEE J. Select Areas Commun., vol. 20, pp. 1211-1226, Aug. 2002.

[62] K. Yu, M. Bengtsson, B. Ottersten, D. McNamara, P. Karlsson, and M. Beach, "A wideband statistical model for NLOS indoor MIMO channels," in Proc. IEEE 55th Veh. Technol. Conf., vol. 1, Birmingham, AL, May 6-9, 2002, pp. 370-374.

[63] H.Özcelik, M. Herdin, W. Weichselberger, J. Wallace, and E. Bonek, "Deficiencies of the kronecker MIMO radio channel model," Electron. Lett., vol. 39, pp. 1209-1210, Aug. 2003.
[64] T. Svantesson and J. W. Wallace, "Tests for assessing multivariate normality and the covariance structure of MIMO data," in Proc. IEEE Int. Conf. Acoustics, Speach, and Signal Processing, vol. 4, Hong Kong, Apr. 6-10, 2003, pp. 656-659.

[65] J. W. Wallace and M. A. Jensen, "Modeling the indoor MIMO wireless channel," IEEE Trans. Antennas Propagat., vol. 50, pp. 591-599, May 2002.

[66] T. D. Abhayapala, T. S. Pollock, and R. A. Kennedy, "Spatial decomposition of MIMO wireless channels," in Proc. 7th Int. Symp. on Signal Processing and it Applications, ISSPA, vol. 1, Paris, France, Jul. 1-4, 2003, pp. 309-312.

[67] M. Steinbauer, A. F. Molisch, and E. Bonek, "The double-directional radio channel," IEEE Antennas Propagat. Mag., vol. 43, pp. 51-63, Aug. 2001.

[68] H. Krim and M. Viberg, "Two decades of array signal processing research: The parametric approach," IEEE Signal Processing Mag., vol. 13, pp. 67-94, July 1996.

[69] Q. H. Spencer, B. D. Jeffs, M. A. Jensen, and A. L. Swindlehurst, "Modeling the statistical time and angle of arrival characteristics of an indoor multipath channel," IEEE J. Select Areas Commun., vol. 18, pp 347-360, Mar. 2000.

[70] J. Fuhl, J.-P. Rossi, and E. Bonek, "High-resolution 3-D direction-of-arrival determination for urban mobile radio," IEEE Trans. Antennas Propagat., vol. 45, pp. 672-682, Apr. 1997.

[71] A. Richter, D. Hampicke, G. Sommerkorn, and R. S. Thoma, "Joint estimation of DoD, time-delay, and DoA for high-resolution channel sounding," in Proc. IEEE 51st Veh. Technol. Conf., vol. 2, Tokyo, Japan, May 15-18, 2000, pp. 1045-1049.

[72] A. F. Molisch, M. Steinbauer, M. Toeltsch, E. Bonek, and R. S. Thoma, "Capacity of MIMO systems based on measured wireless channels," IEEE J. Select Areas Commun., vol. 20, pp. 561-569, Apr. 2002.

[73] T. Kurner, D. J. Cichon, and W. Wiesbeck, "Concepts and results for 3D digital terrain-based wave propagation models: An overview," IEEE J. Select Areas Commun., vol. 11, pp. 1002-1012, Sept. 1993.

[74] H. L. Bertoni, W. Honcharenko, L. R. Macel, and H. H. Xia, "UHF propagation prediction for wireless personal communications," Proc. IEEE, vol. 82, pp. 1333-1359, Sept. 1994.

[75] S. Y. Seidel and T. S. Rappaport, "Site-specific propagation prediction for wireless in-building personal communication system design," in Proc. IEEE 52nd Veh. Technol. Conf., vol. 43, 1994, pp. 879-891.

[76] D. J. Cichon and T. Kurner, "Propagation Prediction Models,", COST 231 Final Rep., 1995.

[77] G. E. Athanasiadou, A. R. Nix, and J. P. McGeehan, "A microcellular ray-tracing propagation model and evaluation of its narrow-band and wide-band predictions," IEEE J. Select Areas Commun., vol. 18, pp. 322-335, Mar. 2000.

[78] M. F. Iskander and Z. Yun, "Propagation prediction models for wireless communication systems," IEEE Trans. Microwave Theory Tech., vol. 50, pp. 662-673, Mar 2002

[79] Y. Wang, S. Safavi-Naeini, and S. K. Chaudhuri, "A hybrid technique based on combining ray tracing and FDTD methods for site-specific modeling of indoor radio wave propagation," IEEE Trans. Antennas Propagat., vol. 48, pp. 743-754, May 2000.

[80] Z. Zhang, R. K. Sorensen, Z. Yun, M. F. Iskander, and J. F. Harvey, "A ray-tracing approach for indoor/outdoor propagation through window structures," IEEE Trans. Antennas Propagat., vol. 50, pp. 742-748, May 2002.

[81] A. L. Swindlehurst, G. German, J. Wallace, and M. Jensen, "Experimental measurements of capacity for MIMO indoor wireless channels," in Proc. IEEE 3rd Workshop on Signal Processing Advances in Wireless Communications (SPAWC '01), Mar. 2001, pp. 30-33.

[82] H. Zhu, J. Takada, and T. Kobayashi, "The verification of a deterministic spatio-temporal channel modeling approach by applying a deconvolution technique in the measurement," in Proc. IEEE 53rd Veh. Technol. Conf., vol. 1, Rhodes, Greece, May 6-9, 2001, pp. 362-366.

[83] A. Muller, "Monte-carlo multipath simulation of ray tracing channel models," in Proc. IEEE Global Telecomm. Conf., vol. 3, San Francisco, CA, Nov.-Dec. 11-2, 1994, pp. 1446-1450.

[84] R. A. Valenzuela, "Ray tracing prediction of indoor radio propagation," in Proc. IEEE 5th Int. Symp. on Personal, Indoor and Mobile Radio Comm., vol. 1, The Hague, Netherlands, Sept. 18-23, 1994, pp. 140-144.

[85] P. Marques, J. Fernandes, and J. Neves, "Complex impulse response modeling for wideband channels," in Proc. IEEE Spring Veh. Technol. Conf., vol. 2, Ottawa, Ontario, Canada, May 18-21, 1998, pp. 702-706. 
[86] K. R. Dandekar, A. Arredondo, G. Xu, and H. Ling, "Using ray tracing to study urban vector channel propagation characteristics," in Proc. IEEE Spring Veh. Technol. Conf., vol. 1, Houston, TX, May 16-20, 1999, pp. 381-385.

[87] F. Tila, P. R. Shepherd, and S. R. Pennock, "Theoretical capacity evaluation of indoor micro- and macro-MIMO systems at $5 \mathrm{GHz}$ using site specific ray tracing," Electronics Letters, vol. 39, pp. 471-472, Mar. 2003.

[88] J.-H. Jo, M. A. Ingram, and N. Jayant, "Angle clustering in indoor spacetime channels based on ray tracing," in Proc. IEEE 54th Veh. Technol. Conf., vol. 4, Atlantic City, NJ, Oct. 7-11, 2001, pp. 2067-2071.

[89] T. Svantesson, "A physical MIMO radio channel model for multi-element multi-polarized antenna systems," in Proc. IEEE 54th Veh. Technol. Conf., vol. 2, Atlantic City, NJ, Oct. 7-11, 2001, pp. 1083-1087.

[90] — "A double-bounce channel model for multi-polarized MIMO systems," in Proc. IEEE 56th Veh. Technol. Conf., vol. 2, Vancouver, BC, Sep. 24-28, 2002, pp. 691-695.

[91] L. Hanlen and M. Fu, "Multiple antenna wireless communication systems: Limits to capacity growth," in Proc. IEEE Wireless Comm. and Networking Conf., vol. 1, Mar. 17-21, 2002, pp. 172-176.

[92] R. Tingley and K. Bahlavan, "A statistical model of space-time radio propagation in indoor environments," in Proc. IEEE-APS Conf. on Antennas and Propagation for Wireless Comm., Waltham, MA, Nov. 6-8, 2000, pp. 61-64.

[93] A. F. Molisch, "A generic model for MIMO wireless propagation channels," in Proc. IEEE Int. Conf. Commun., vol. 1, New York, NY, Apr.-May 28-2, 2002, pp. 277-282.

[94] _ "A channel model for MIMO systems in macro- and microcellular environments," in Proc. IEEE 55th Veh. Technol. Conf., vol. 2, Birmingham, AL, May 6-9, 2002, pp. 655-659.

[95] R. J. Piechocki, J. P. McGeehan, and G. V. Tsoulos, "A new stochastic spatio-temporal propagation model (SSTPM) for mobile communications with antenna arrays," IEEE Trans. Commun., vol. 49, pp. 855-862, May 2001.

[96] C. Oestges, V. Erceg, and A. J. Paulraj, "A physical scattering model for MIMO macrocellular broadband wireless channels," IEEE J. Select Areas Commun., vol. 21, pp. 721-729, Jun. 2003.

[97] R. B. Ertel and J. H. Reed, "Angle and time of arrival statistics for circular and elliptical scattering models," IEEE J. Select Areas Commun., vol. 17, pp. 1829-1840, Nov. 1999.

[98] A. Abdi and M. Kaveh, "A space-time correlation model for multielement antenna systems in mobile fading channels," IEEE J. Select Areas Commun., vol. 20, pp. 550-560, Apr. 2002.

[99] J. C. Liberti and T. S. Rappaport, "A geometrically based model for line-of-sight multipath radio channels," in Proc. 1996 IEEE Spring Veh. Technol. Conf., vol. 2, Atlanta, GA, Apr.-May 28-1, 1996, pp. 844-848.

[100] O. Norklit and J. B. Andersen, "Diffuse channel model and experimental results for array antennas in mobile environments," IEEE Trans. Antennas Propagat., vol. 46, pp. 834-840, Jun. 1998.

[101] G. L. Turin, F. D. Clapp, T. L. Johnston, B. F. Stephen, and D. Lavry, "A statistical model of urban multipath propagation," IEEE Trans. Veh. Technol., vol. VT-21, pp. 1-9, Feb. 1972.

[102] A. A. M. Saleh and R. A. Valenzuela, "A statistical model for indoor multipath propagation," IEEE J. Select Areas Commun., vol. SAC-5, pp. 128-137, Feb. 1987.

[103] C.-C. Chong, C.-M. Tan, D. I. Laurenson, S. McLaughlin, M. A. Beach, and A. R. Nix, "A new statistical wideband spatio-temporal channel model for 5-GHz band WLAN systems," IEEE J. Select Areas Commun. vol. 21, pp. 139-150, Feb. 2003.

[104] T. Zwick, C. Fischer, D. Didascalou, and W. Wiesbeck, "A stochastic spatial channel model based on wave-propagation modeling," IEEE J. Select Areas Commun., vol. 18, pp. 6-15, Jan. 2000.

[105] R. J. C. Bultitude, S. A. Mahmoud, and W. A. Sullivan, "A comparison of indoor radio propagation characteristics at $910 \mathrm{MHz}$ and $1.75 \mathrm{GHz}$," IEEE J. Select Areas Commun., vol. 7, pp. 20-30, Jan. 1989.

[106] R. Ganesh and K. Pahlavan, "Statistical modeling and computer simulation of indoor radio channel," Proc. Inst. Elect. Eng. Part 1: Communications, Speech and Vision, vol. 138, pp. 153-161, June 1991.

[107] J.-G. Wang, A. S. Mohan, and T. A. Aubrey, "Angles-of-arrival of multipath signals in indoor environments," in Proc. IEEE Spring Veh. Technol. Conf., vol. 1, Atlanta, GA, Apr.-May 28-1, 1996, pp. 155-159.

[108] L. M. Correia, Wireless Flexible Personalised Communications: Wiley, 2001.

[109] N. Kita, S. Uwano, and A. Sato, "New multipath propagation model of high-speed wireless access system for residential area using $5-\mathrm{GHz}$ band," in Proc. IEEE 54th Veh. Technol. Conf., vol. 3, Atlantic City, NJ, Oct. 7-11, 2001, pp. 1621-1625.
[110] J. Fuhl, A. F. Molisch, and E. Bonek, "Unified channel model for mobile radio systems with smart antennas," in Proc. Inst. Elect. Eng. Radar, Sonar and Navigation, vol. 145, Feb. 1998, pp. 32-41.

[111] M. Stege, J. Jelitto, M. Bronzel, and G. Fettweis, "A multiple input-multiple output channel model for simulation of Tx- and Rx-diversity wireless systems," in Proc. IEEE 52nd Veh. Technol. Conf., vol. 2, Boston, MA, Sept. 24-28, 2000, pp. 833-839.

[112] D. Chizhik, G. J. Foschini, M. J. Gans, and R. A. Valenzuela, "Propagation and capacities of multi-element transmit and receive antennas," in Proc. IEEE Antennas and Propagat. Society Int. Symp., vol. 1, Boston, MA, July 8-13, 2001, pp. 438-441.

[113] — - "Keyholes, correlations, and capacities of multielement transmit and receive antennas," IEEE Trans. Wireless Commun., vol. 2, pp. 361-368, Apr. 2002

[114] P. Almers, F. Tufvesson, and A. F. Molisch, "Measurement of keyhole effect in a wireless multiple-input multiple-output (MIMO) channel," IEEE Commun. Lett., vol. 7, pp. 373-375, Aug. 2003.

[115] R. G. Vaughan and J. B. Andersen, "Antenna diversity in mobile communications," IEEE Trans. Veh. Technol., vol. VT-36, pp. 147-172, Nov. 1987.

[116] T. Svantesson, "Correlation and channel capacity of MIMO systems employing multimode antennas," IEEE Trans. Veh. Technol., vol. 51, pp. 1304-1312, Nov. 2002.

[117] C. Waldschmidt, T. Fugen, and W. Wiesbeck, "Spiral and dipole antennas for indoor MIMO-systems," IEEE Antennas Wireless Propagat. Lett., vol. 1, no. 1, pp. 176-178, 2002.

[118] S. Sandhu, R. U. Nabar, D. A. Gore, and A. Paulraj, "Near-optimal selection of transmit antennas for a MIMO channel based on Shannon capacity," in Proc. 34th Asilomar Conf. Signals, Systems and Computers, vol. 1, Pacific Grove, CA, Oct.-Nov. 29-1, 2000, pp. 567-571.

[119] D. Gore and A. Paulraj, "Space-time block coding with optimal antenna selection," in Proc. IEEE Int. Conf. Acoustics, Speach, and Signal Processing, vol. 4, Salt Lake City, UT, May 7-11, 2001, pp. 2441-2444.

[120] D. A. Gore, R. W. Heath, and A. J. Paulraj, "Transmit selection in spatial multiplexing systems," IEEE Commun. Lett., vol. 6, pp. 491-493, Nov. 2002.

[121] D. A. Gore and A. J. Paulraj, "MIMO antenna subset selection with space-time coding," IEEE Trans. Signal Processing, vol. 50, pp. 2580-2588, Oct. 2002.

[122] A. F. Molisch, J. H. Winters, and M. Z. Win, "Capacity of MIMO systems with antenna selection," in Proc. IEEE Int. Conf. Commun., vol. 2, Helsinki, Finland, June 11-14, 2001, pp. 570-574.

[123] R. W. Heath Jr., S. Sandhu, and A. Paulraj, "Antenna selection for spatial multiplexing systems with linear receivers," IEEE Commun. Lett., vol. 5, pp. 142-144, Apr. 2001

[124] J. W. Wallace and M. A. Jensen, "Intrinsic capacity of the MIMO wireless channel," in Proc. IEEE 56th Veh. Technol. Conf., vol. 2, Vancouver, British Columbia, Canada, Sep. 24-28, 2002, pp. 701-705.

[125] R. A. Andrews, P. P. Mitra, and R. deCarvalho, "Tripling the capacity of wireless communications using electromagnetic polarization," Nature, vol. 409, pp. 316-318, Jan. 2001.

[126] T. Svantesson, "On capacity and correlation of multi-antenna systems employing multiple polarizations," in Proc. IEEE Antennas and Propagation Society Int. Symp., vol. 3, San Antonio, TX, June 16-21, 2002, pp. 202-205.

[127] T. Svantesson, M. A. Jensen, and J. W. Wallace, "Analysis of electromagnetic field polarizations in multi-antenna systems," IEEE Trans. Wireless Commun., vol. 3, Mar. 2004.

[128] J. B. Andersen and B. N. Getu, "The MIMO cube - A compact MIMO antenna," in Proc. 5th Int. Symp. on Wireless Personal Multimedia Communications, vol. 1, Honolulu, HI, Oct. 27-30, 2002, pp. 112-114.

[129] R. G. Vaughan, "Polarization diversity in mobile communications," IEEE Trans. Veh. Technol., vol. 39, pp. 177-186, Aug. 1990.

[130] P. Kyritsi and D. C. Cox, "Propagation characteristics of horizontally and vertically polarized electric fields in an indoor environment: Simple model and results," in Proc. IEEE 54th Veh. Technol. Conf., vol. 3, Atlantic City, NJ, Oct. 7-11, 2001, pp. 1422-1426.

[131] P. Soma, D. S. Baum, V. Erceg, R. Krishnamoorthy, and A. J. Paulraj, "Analysis and modeling of multiple-input multiple-output (MIMO) radio channel based on outdoor measurements conducted at $2.5 \mathrm{GHz}$ for fixed BWA applications," in Proc. IEEE Int. Conf. Commun., vol. 1, New York, Apr.-May 28-2, 2002, pp. 272-276.

[132] P. Kyritsi, D. C. Cox, R. A. Valenzuela, and P. W. Wolniansky, "Effect of antenna polarization on the capacity of a multiple element system in an indoor environment," IEEE J. Select Areas Commun., vol. 20, pp. 1227-1239, Aug. 2002. 
[133] H. Bolcskei, R. U. Nabar, V. Erceg, D. Gesbert, and A. J. Paulraj, "Performance of spatial multiplexing in the presence of polarization diversity," in Proc. IEEE Int. Conf. Acoustics, Speach, and Signal Processing, vol. 4, Salt Lake City, UT, May 7-11, 2001, pp. 2437-2440.

[134] J. L. Allen and B. L. Diamond, "Mutual Coupling in Array Antennas," Lincoln Laboratory, M.I.T., Tech. Rep. Technical Rep. 424 (ESD-TR-66-443), 1966.

[135] C. A. Balanis, Antenna Theory: Analysis and Design. New York: Wiley, 1997.

[136] J. Luo, J. R. Zeidler, and S. McLaughlin, "Performance analysis of compact antenna arrays with MRC in correlated nakagami fading channels," IEEE Trans. Veh. Technol., vol. 50, pp. 267-277, Jan. 2001.

[137] T. Miki and M. Hata, "Performance of $16 \mathrm{kbit} / \mathrm{s}$ gmsk transmitter with postdetection selection diversity in land mobile radio," IEEE Trans. Veh. Technol., vol. VT-33, pp. 128-133, Aug. 1984.

[138] T. Svantesson and A. Ranheim, "Mutual coupling effects on the capacity of multielement antenna systems," in Proc. IEEE Int. Conf. Acoustics, Speach, and Signal Processing, vol. 4, Salt Lake City, UT, May 7-11, 2001, pp. 2485-2488.

[139] M. Stoytchev, H. Safar, A. L. Moustakas, and S. Simon, "Compact antenna arrays for MIMO applications," in Proc. IEEE Antennas and Propagation Society Int. Symp., vol. 3, Boston, MA, July 8-13, 2001, pp. 708-711.

[140] C. Waldschmidt, J. v. Hagen, and W. Wiesbeck, "Influence and modeling of mutual coupling in MIMO and diversity systems," in Proc. IEEE Antennas and Propagation Society Int. Symp., vol. 3, San Antonio, TX, June 16-21, 2002, pp. 190-193.

[141] M. C. Leifer, "Signal correlations in coupled cell and MIMO antennas," in Proc. IEEE Antennas and Propagation Society Int. Symp., vol. 3, San Antonio, TX, June 16-21, 2002, pp. 194-197.

[142] R. R. Ramirez and F. De Flaviis, "A mutual coupling study of linear polarized microstrip antennas for use in BLAST wireless communications architecture," in Proc. IEEE Antennas and Propagation Society Int. Symp., vol. 2, Salt Lake City, UT, July 16-21, 2000, pp. 490-493.

[143] J. W. Wallace and M. A. Jensen, "The capacity of MIMO wireless systems with mutual coupling," in Proc. IEEE 56th Veh. Technol. Conf., vol. 2, Vancouver, British Columbia, Canada, Sep. 24-28, 2002, pp. 696-700.

[144] - "Termination-dependent diversity performance of coupled antennas: Network theory analysis," IEEE Trans. Antennas Propagat., vol. 52, pp. 98-105, Jan. 2004.

[145] R. G. Vaughan and N. L. Scott, "Closely spaced monopoles for mobile communications," Radio Sci., vol. 28, pp. 1259-1266, Nov.-Dec. 1993.

[146] R. Janaswamy, "Effect of element mutual coupling on the capacity of fixed length linear arrays," IEEE Antennas Wireless Propagat. Lett., vol. 1, no. 1, pp. 157-160, 2002.

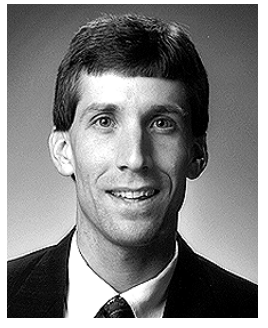

Michael A. Jensen (S'93-M'95-SM'01) received the B.S. (summa cum laude) and M.S. degrees in electrical engineering from Brigham Young University (BYU), Provo, UT, in 1990 and 1991, respectively, and the Ph.D. in electrical engineering from the University of California, Los Angeles (UCLA), in 1994.

From 1989 to 1991, he was a Graduate Research Assistant in the Lasers and Optics Laboratory, BYU. From 1991 to 1994, he was a Graduate Student Researcher in the Antenna Laboratory, UCLA. Since 1994, he has been at the Electrical and Computer Engineering Department, BYU, where he is currently an Associate Professor. His main research interests include antennas and propagation for personal communications, microwave circuit design, radar remote sensing, numerical electromagnetics, and optical fiber communications.

Dr. Jensen is a Member of Eta Kappa Nu and Tau Beta Pi. In 1990, he received a National Science Foundation Graduate Fellowship. He was awarded the best student paper award at the 1994 IEEE International Symposium on Antennas and Propagation, and received the Harold A. Wheeler Applications Prize Paper Award in the IEEE TRANSACTIONS ON ANTENNAS AND PROPAGATION in 2002. He currently serves on the Joint Meetings Committee for the IEEE Antennas and Propagation Society and as an Associate Editor for the IEEE TRANSACTIONS ON ANTENNAS AND PROPAGation. He was the Technical Program Chair for the 2000 IEEE International Symposium on Antennas and Propagation and the General Co-Chair for the IEEE Topical Conference on Wireless Communications in 2003.

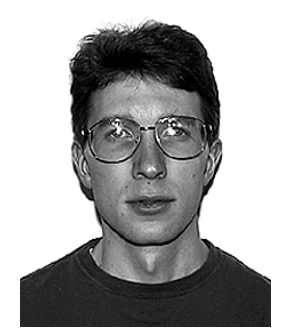

Jon W. Wallace (S'99-M'03) received the B.S. (summa cum laude) and $\mathrm{Ph} . \mathrm{D}$. degrees in electrical engineering from Brigham Young University (BYU), Provo, UT, in 1997 and 2002, respectively.

From 1995 to 1997, he worked as an Associate of Novell, Incorporated, Provo, UT. During 1997, he was a Member of Technical Staff for Lucent Technologies, Denver, CO. From 1998 to 2002, he worked as a Graduate Student Researcher in the BYU Wireless Communications Laboratory where he is currently a Research Associate. From 2002 to 2003, he visited the Technical University of Vienna Mobile Communications Group. His research interests include wireless channel sounding and modeling, optical device modeling, and remote sensing.

Dr. Wallace received the National Science Foundation Graduate Fellowship in 199 and the Harold A. Wheeler Applications Prize Paper Award in theIEEE TRANSACTIONS ON ANTENNAS AND PROPAGATION in 2002. 